\title{
New Concept of PLC Modems: Multi-Carrier System for Frequency Selective Slow-Fading Channels Based on Layered SCCC Turbocodes
}

\author{
Jan ZAVRTÁLEK, Daniel KEKRT, Jaromír HRAD
}

Dept. of Telecomm. Engineering, Czech Technical University in Prague, Technická 2, 16627 Praha, Czech Republic

zavrtjan, kekrtd1, hrad@fel.cvut.cz

\begin{abstract}
The article introduces a novel concept of a PLC modem as a complement to the existing G3 and PRIME standards for communications using medium- or high-voltage overhead or cable lines. The proposed concept is based on the fact that the levels of impulse noise and frequency selectivity are lower on high-voltage lines than on low-voltage ones. Also, the demands for "cost-effective" circuitry design are not so crucial as in the case of modems for low-voltage level. In contract to these positive conditions, however, there is the need to overcome much longer distances and to take into account low SNR on the receiving side. With respect to the listed reasons, our concept makes use of MCM, instead of OFDM. The assumption of low SNR is compensated through the use of an efficient channel coding based on a serially concatenated turbo code. In addition, MCM offers lower latency and PAPR compared to OFDM. Therefore, when using $M C M$, it is possible to excite the line with higher power. The proposed concept has been verified during experimental transmission of testing data over a real, $5 \mathrm{~km}$ long, $22 \mathrm{kV}$ overhead line.
\end{abstract}

\section{Keywords}

Power line communication, multi-carrier modulation, serially concatenated convolutional codes, iterative detection, BCJR forward-backward algorithm, softoutput Viterbi algorithm, soft-in soft-out module, expectation-maximization algorithm, soft decision directed synchronization, data aided synchronization, joint iterative synchronization and detection

\section{Introduction}

Reliable and fast power line communication (PLC) is a key prerequisite for successful deployment and operation of Smart Grid networks. At present, only the communication over low-voltage power lines is largely used, thanks to relatively inexpensive hardware, mainly for Automatic Meter Reading (AMR), tariffs and load management (Automatic Meter Management, AMM), and complex monitoring of network infrastructure (Advanced Metering Infrastructure, AMI) in order to increase the reliability of power distribution, which is the last step on the way towards a Smart Grid. Networks are usually controlled from substations and distribution stations, using (1) medium- or high-voltage power line, (2) dedicated data link (Internet, dedicated data circuit, optical cable, etc.). It is only logical to use the existing medium-voltage power lines for data communication, thus eliminating the need of a dedicated data link. Such solution can reduce the dependence on external factors and thereby increase the reliability of power distribution networks. Due to rapid deployment of so-called renewable energy sources, the reliability and stability of the network is becoming a serious problem.

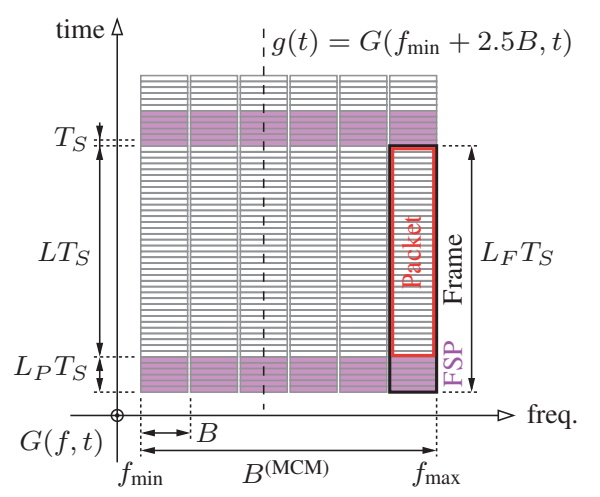

Fig. 1. Time-frequency partition of packets for serial MCM.

In this article we propose a novel communication system, designed specifically for communication over mediumvoltage (mv) power lines. Its purpose is to fill the gap in the existing systems based on OFDM (like G.hnem, IEEE P1901.2, G3, PRIME), since their robustness is somewhat lower when they are used for long-distance transmission over medium-voltage power lines.

The architecture of the proposed solution is based on the assumed physical characteristics of the transmission media (i.e. medium-voltage overhead or cable lines) that feature moderate frequency selectivity within CENELEC A band and high levels of noise. The type of the noise, in general, is AWGN (Additive White Gaussian Noise). In such 


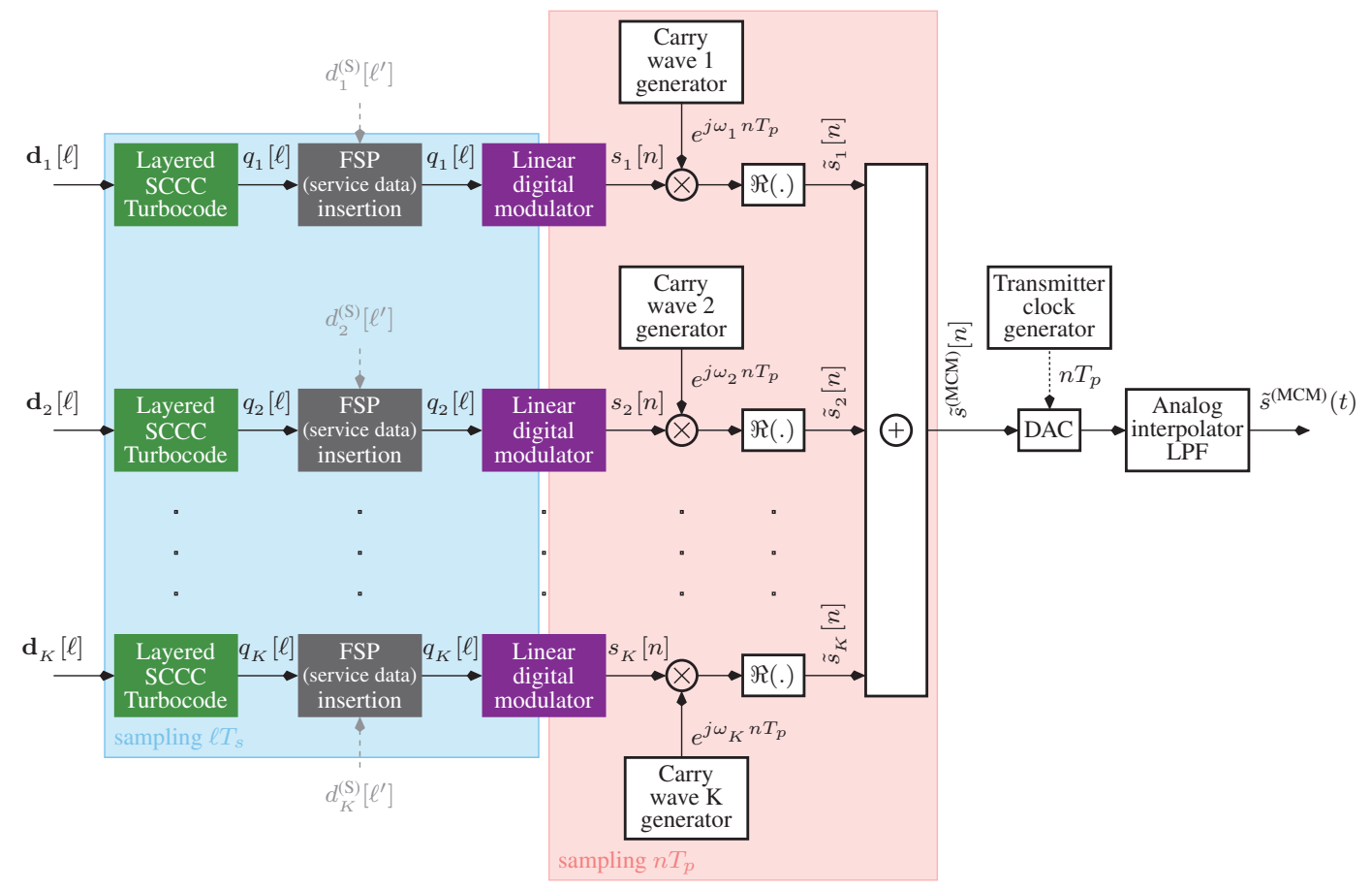

Fig. 2. Block diagram of a transmitter for serial MCM.

case, the OFDM (Orthogonal Frequency Division Multiplexing) technology is not suitable because of its high PAPR (Peak-to-Average Power Ratio) - it should be used rather for channels with high frequency selectivity, or significant multipath signal propagation. Moreover, OFDM introduces high processing latency in the receiving digital front-end, as it uses FFT and IFFT integral transforms. The latency can be partially reduced by shortening of the OFDM frame; however, it results in lower spectral efficiency (number of carriers). Another technology to be considered is wideband single-carrier linear digital modulation, which has low PAPR, but it is not immune against frequency selectivity. In a frequency-selective channel, it requires equalization at the receiving side, computationally intensive channel estimation and a complicated core for channel inversion. For these reasons we propose MCM (Multi-Carrier Modulation) as the optimum technology for data communication over mv power lines, i.e., a set of subchannels using narrowband linear digital modulations, with equidistantly or non-equidistantly spaced carriers over the given bandwidth. Such approach offers sufficiently low PAPR, and also very low processing latency (only matched filtering is performed on the receiving side). Every subchannel is considered as frequency-flat, and therefore no equalization is needed - only simple synchronization of amplitude, phase and symbol timing is required.

The paper is organized as follows. Section 2 describes Serial MCM transmission. Sections 3 and 4 describes MCM Transmitter and Receiver. Section 5 describes experimental measurement. Finally, Section 6 presents conclusions and ideas for future work.

\section{Serial MCM Transmission}

The proposed architecture of MCM PLC modem is based on serial data transmission. The individual subchannels are entirely independent, carrying separate data communications at lower bitrate, corresponding to the subchannel bandwidth. If SNR in some of the subchannels is negatively affected by strong noise, interference or destructive deformation (gap) in channel frequency response, the data is directed to other subchannels where the conditions are better. So, the concept is based on the assumption that transmission conditions are not bad across the entire MCM band.

The individual data packets are split into subchannels in time-frequency domain as described in Fig. 1, and their transmission may but need not be entirely independent. FSP (Frame Synchronization Preamble) stands for synchronization preamble of a frame containing $L_{P}$ channel symbols, $T_{S}$ is symbol period, $L$ and $L_{F}$ mean packet length and frame length, respectively, expressed as the number of channel symbol, $B$ and $B^{(\mathrm{MCM})}$ mean subchannel bandwidth and total modulation bandwidth, respectively, and $G(f, t)$ is timefrequency transfer function of the channel.

\section{Transmitter Description}

Block diagram of the transmitting side of the proposed MCM PLC modem is shown in Fig. 2. The transmitter consists of two fundamental blocks: multilayer channel coder and digital front-end. 


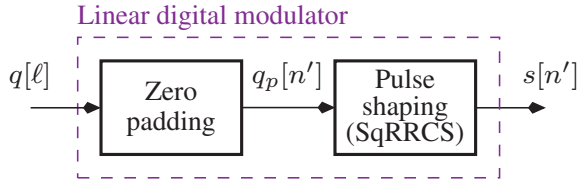

Fig. 3. Block diagram of a linear digital modulator.

\subsection{Transmitter Digital Front-End}

Every branch of the structure represents one subchannel of the resulting composite spectrum. Each particular section of the resulting spectrum is shaped in a narrowband linear digital modulator through a complex filter with real SqRRCS (Square Root Raised Cosine Spectrum) impulse response, as shown in Fig. 3.

\subsection{Turbo Encoders}

Noise immunity is achieved through channel coding based on punctured serial concatenated turbo code, see Fig. 4. At present, it is one of the most efficient channel coding techniques. Its coding gain grows with the length of the encoded packet. This is the only negative property of turbo coding, as it implies limitation for transmission of short packets; suitable packet lengths are 1024 or more symbols. More information about turbo codes can be found in [1]-[4].

For the proposed concept we have chosen a proven architecture, in which the outer FSM (Finite State Machine) is based on a simple systematic forward- or backwardrecursive convolution code. The FSM has 2 or 4 internal states. The fixed code rate is $1 / 2$, i.e. the coder adds one protective (redundant) bit to each bit of the information. So, there is a binary data sequence $d[\ell]$ at the input of the coder, and another sequence $c[\ell]$ at its output, in modulo 4 arithmetic. The sequence $c[\ell]$ is taken to the permutator I and then passed to the inner FSM. The inner FSM is based on a simple integrator with 4 internal states and $1 / 1$ rate. The fixed turbo code rate is $1 / 2$, and the code symbols $c^{(\mathrm{TC})}[\ell]$ at the output can be directly mapped to the QPSK constellation, with respect to Ungerboeck mapping rules.

If the transmission conditions are good and SNR (Signal-to-Noise Ratio) high, then the modem switches to layered turbo code mode (see Fig. 5), in which the data are transmitted in 2, 3 or 4 streams hierarchically mapped to 16QAM, 64QAM or 256QAM, and $K$ equals to 4 . Thanks to the hierarchical mapping, the individual data streams have different priorities: stream \#1 has the highest priority, while stream \#4 the lowest one. The reason is that stream \#1 is mapped to the highest-quality points of QAM constellation (i.e. those with the largest mutual Euclidean distances). Therefore the noise immunity of stream \#1 will be very high, at the expense of stream \#4 that is mapped to the lowestquality points, thus having low noise immunity. If all data streams are required to behave equally, a commutator is prepended to the mapper input; then hierarchical mapping will be rotated over the time, and so the respective noise immunities of the individual streams will be equalized. Besides the hierarchical mapping, the proposed concept allows also modification of the initial code rate using the punctured mapping. Suitable puncturing changes the initial code rate $1 / 2$ to the output code rates $2 / 3$ and $3 / 4$. When the SNR is low, the transmitter uses the default code rate $1 / 2$. As the SNR grows, the transmitter is switched firstly to the rate $2 / 3$, and subsequently to $3 / 4$. Code rates of all considered modem configurations along with the assumed SNR requirements are summarized in Tab. 1. The overview refers to MCM with total bandwidth of $80 \mathrm{kHz}$ consisting of 8 subbands, $9.5 \mathrm{kHz}$ each, separated by $0.5 \mathrm{kHz}$ gaps; the centers of the individual channels are at $40 \mathrm{kHz}, 50 \mathrm{kHz}, 60 \mathrm{kHz}, \ldots, 110 \mathrm{kHz}$.

\section{Receiver Description}

Block diagram of the receiving side is shown in Fig. 6. The receiver consists of three fundamental blocks: digital front-end, multilayer frame synchronization block, and a set of joint iterative synchronizers-detectors allocated through a switch.

\subsection{Receiver Digital Front-End}

The individual subchannels are firstly mixed with the corresponding subcarriers to obtain a set of signals (complex envelopes) in baseband. Then these baseband signals are decimated in a down-sampling cascade consisting of a series of anti-aliasing filters and decimators (CIC filter). At its output we get sub-sampled complex envelopes $r_{k}\left[n^{\prime}\right]$ so that there are 4 samples per or one symbol period, which is perfectly in accord with the sampling theorem. The particular sub-sampled complex envelopes are then shaped and partially rid of AWGN in matched filters with real impulse response SqRRCS. Consolidated complex envelopes at the output of the matched filters $x_{k}\left[n^{\prime}\right]$ are then passed to a set of frame synchronizers.

\subsection{Frame Synchronization}

Frame synchronizers search for synchronization preambles in the individual streams, which are used to localize the beginnings of particular packets (frame synchronization), and also for initial estimates of subchannel attenuation $\left|\hat{g}_{k}^{(0)}\right|$, phase rotation $\angle \hat{g}_{k}^{(0)}$, symbol timing $\hat{\tau}_{k}^{(0)}$ and standard deviation of AWGN $\hat{\sigma}_{w_{k}}^{(0)}$. These values are estimated using Data Aided (DA) Maximum-Likelihood (ML) Channel State Estimators (CSE), which are autonomous functional sub-blocks of the frame synchronizers.

The proposal assumes that one of the following two types of synchronization preambles (training sequences) should be used - Legender or Rudin-Shapiro (R-S). The length of Legender sequences can be 11, 19 or 31 bits (without cyclic prefix). These lengths were selected because of their sequence correlation properties. By adding the cyclic 


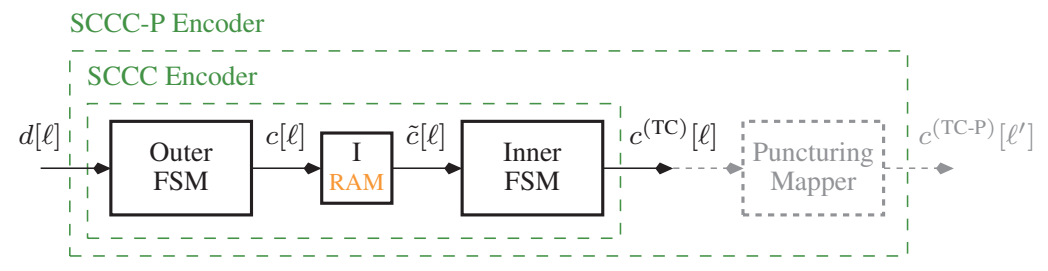

Fig. 4. Block diagram of a punctured serial turbo coder.

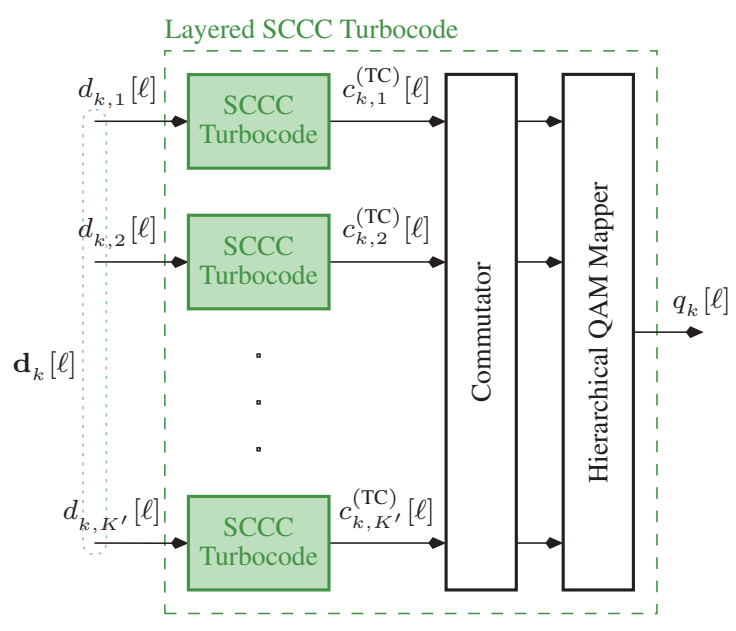

Fig. 5. Block diagram of a layered serial turbo coder.

prefix, the lengths can be extended to even bits count suitable for practical implementation. The second option is to use a pair of R-S preambles, which can be used to transmit a very slow, but very robust binary stream called service data. The principle of transmitting service data is quite simple. Let us select one R-S preamble from the pair, according to the value of the input bit $d_{k}^{(\mathrm{S})}\left[\ell^{\prime}\right]$. On the receiving side, so-called dual version of frame synchronizer is then used, which searches for both of the R-S preambles. Its output is $\hat{d}_{k}^{(S)}\left[\ell^{\prime}\right]$ estimate depending on the detected R-S preamble. The bitrate of service data depends on the length of a packet and of the used R-S preambles including the cyclic prefix see Tab. 1. The default lengths of these training sequence pairs are $2^{l}$, where $l=1,2,3, \ldots, N$.

\subsection{Joint Iterative Synchronizer-Decoder}

Samples of complex envelope, isolated by frame synchronizers and corresponding to one data packet in each subchannel, are passed through the switch to the joint iterative synchronizer-detector - see Fig. 7. Together with this section, the initial estimates of $\hat{g}_{k}^{(0)}, \hat{\tau}_{k}^{(0)}$ and $\hat{\sigma}_{w_{k}}^{(0)}$ are handed over. Processing of the complex envelope begins in so-called Soft Output Demodulator (SODEM) shown in Fig. 8. Firstly, the complete envelope section is stored into RAM memory of the demodulator. Then it is resampled and decimated in Decimator-Interpolator (DFA) based on Farrow algorithm. The resampling is controlled by symbol timing estimate $\hat{\tau}^{(I)}$. At the DFA output we get time-synchronized sequence of channel symbols $x_{h}[\ell]$. These symbols are then corrected in amplitude and phase, depending on channel estimate $\hat{g}^{(I)}$ in order to obtain fully synchronized sequence of channel symbols $x_{h}[\ell]$. The differences between received channel symbols $x_{h}[\ell]$ and transmitted symbols $q[\ell]$ are then caused only by superimposed AWGN noise; now the logarithmic likelihood function or forward detector metrics $\left\{\hat{\mathcal{M}}_{F}^{(I)}\right\}$ (discrete probability density) can be computed, with respect to the estimate of AWGN standard deviation $\hat{\sigma}_{w}^{(I)}$.

The forward metrics of $\hat{\mathcal{M}}_{F}^{(I)}\left(\check{q}_{k}\right)$ proceed into divider/combiner logic of so-called soft inversion of Hierarchic QAM Mapper, the output of which provides composite (layered) forward metrics $\hat{\mathcal{M}}_{F}^{(I)}\left(\check{c}_{k, k^{\prime}}^{(\mathrm{TC})}\right)$. These metrics are used as an input for the individual Iterative Decoding Networks (IDN) of the Serially Concatenated Convolutional Codes (SCCC) - see Fig. 9. The arrangement of the decoding network is equivalent to the structure of turbo coder. So, if punctured turbo code is used on the transmitting side, then the corresponding IDN at the input is prepended by soft inversion of the puncturing mapping block SOM (Soft-Output Mapper). The fundamental components of SCCC IDN are Soft-In Soft-Out (SISO) soft inversion blocks of convolution coders FSM on the transmitting side. The SISO modules perform Forward-Backward Algorithm (FBA), also known as BCJR (Bahl, Cocke, Jelinek and Raviv). More information about BCJR algorithm and its derivatives can be found in [1]-[3] and [5]-[8]. A two-dimensional BCJR algorithm can be found in [12].

The outputs of the particular detection layers are packet data estimates $\hat{d}_{k, k^{\prime}}[\ell]$ and partial (layer) backward metrics $\hat{\mathcal{M}}_{B}^{(I)}\left(\check{c}_{k, k^{\prime}}^{(\mathrm{TC})}\right)$. The hard data estimate $\hat{d}_{k, k^{\prime}}[\ell]$ is computed in the Decision block (DEC) by thresholding of output forward metrics $\hat{\mathcal{M}}_{F}^{(I)}\left(\check{d}_{k}\right)$. The backward partial metrics $\hat{\mathcal{M}}_{B}^{(I)}\left(\check{c}_{k, k^{\prime}}^{(\mathrm{TC})}\right)$ are then combined into backward metrics $\hat{\mathcal{M}}_{B}^{(I)}\left(\check{q}_{k}\right)$ that are immediately transformed, together with the forward metrics $\hat{\mathcal{M}}_{F}^{(I)}\left(\check{q}_{k}\right)$, into posterior probabilities $\hat{\mathcal{P}}^{(I)}$. The resulting sequence of $\hat{\mathcal{P}}^{(I)}$ controls the SDD EM CSE estimator that improves the initial estimates of $\hat{g}_{k}^{(0)}, \hat{\tau}_{k}^{(0)}$ and $\hat{\sigma}_{w_{k}}^{(0)}$.

In this concept it is based on the ExpectationMaximization (EM) criterion, Soft Decision Directed (SDD) by $\hat{\mathcal{P}}^{(I)}\left(\check{q}_{k}\right)$ sequence. The advantage of this estimator is that its kernel is very similar to DA ML CSE blocks in Frame synchronizers. This can be useful for FPGA (Field Programmable Gate Array) implementations - only one fast multiplexed core is sufficient for the synchronizer, identical 


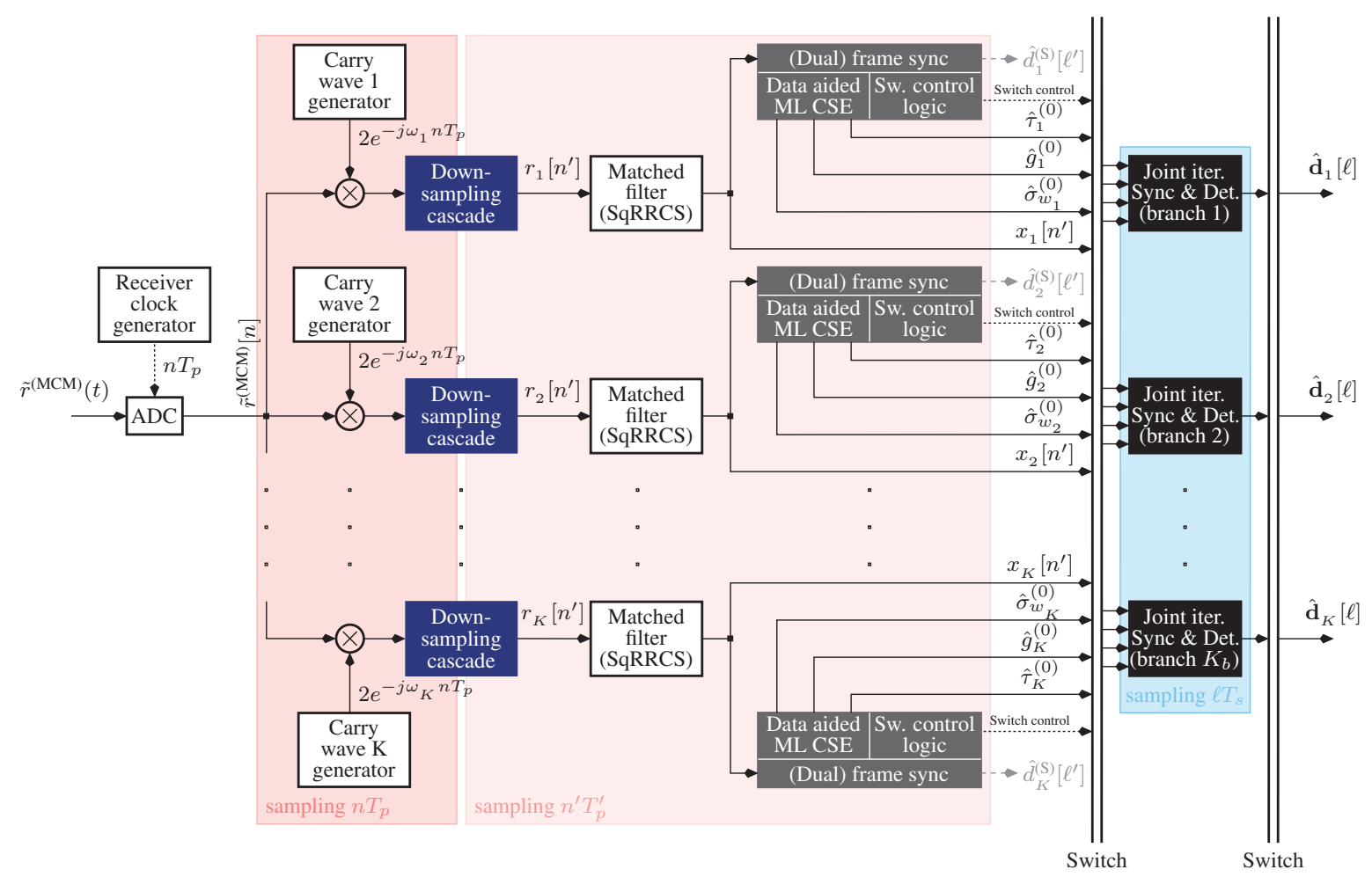

Fig. 6. Block diagram of a receiver using serial MCM.

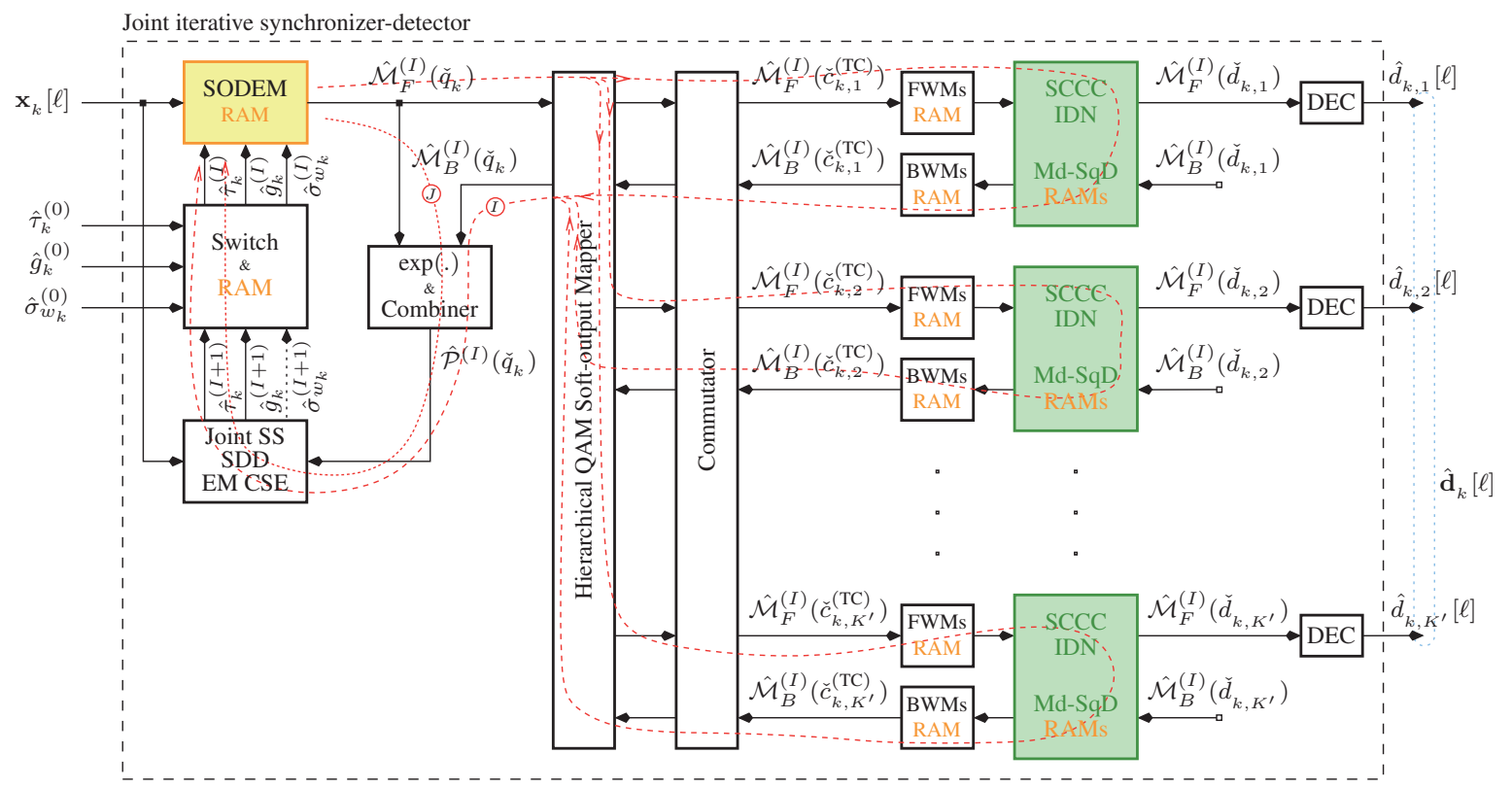

Fig. 7. Block diagram of the layered synchronizer-detector.

for synchronization acquisition mode and for receiver tracking mode. Detailed description of the EM algorithm providing basic synchronization of the iterative decoding network can be found in [17] and [20]. More variants of EM and its derivatives are discussed in [14]-[16] and [18]. Another approach to decoding network synchronization based on socalled adaptive SISO modules, can be found in [19].
The updated estimates of $\hat{g}_{k}^{(I)}, \hat{\tau}_{k}^{(I)}$ and $\hat{\sigma}_{w_{k}}^{(I)}$ are redirected into the Soft Demodulator, and so one iteration $I$ of the system is completed. In the next iteration the whole detection process is repeated with the updated estimates. After performing the given maximum number of iterations or after stabilization of the iterative network, the estimate $\hat{\mathbf{d}}_{k}[\ell]$ at the output of the structure is considered to be the valid data and 


\begin{tabular}{|c|c|c|c|c|c|c|c|c|}
\hline $\begin{array}{l}\text { System } \\
\text { variant }\end{array}$ & $\begin{array}{r}\text { Packet } \\
\text { length } \\
\text { [bits] }\end{array}$ & $\begin{array}{c}\text { Preamble } \\
\text { length } \\
\text { [bits] }\end{array}$ & Alphabet & $\begin{array}{c}\text { Subchannel } \\
\text { bitrate } \\
{[\mathrm{kbit} / \mathrm{s}]}\end{array}$ & $\begin{array}{l}\text { Modulation } \\
\text { bitrate } \\
\text { [kbit/s] }\end{array}$ & $\begin{array}{c}\text { User data } \\
\text { bitrate } \\
{[\mathrm{kbit} / \mathrm{s}]}\end{array}$ & $\begin{array}{c}\text { Service data } \\
\text { bitrate } \\
\text { [bit/s] }\end{array}$ & Usage \\
\hline \multirow[b]{4}{*}{ rate $1 / 2,2 / 3,3 / 4$} & 1024 & 40 & QPSK & \multirow{4}{*}{$\begin{array}{l}8.44, \\
11.25, \\
12.66\end{array}$} & \multirow{4}{*}{$\begin{array}{l}67.55, \\
90.06, \\
101.32\end{array}$} & $65.01,86.68,97.52$ & 63.50 & \multirow{4}{*}{$\begin{array}{c}\text { Very low } \\
\text { SNR }>4 \mathrm{~dB}\end{array}$} \\
\hline & 2048 & 40 & QPSK & & & $66.26,88.34,99.39$ & 32.40 & \\
\hline & 4096 & 40 & QPSK & & & $66.90,89.20,100.35$ & 16.30 & \\
\hline & 8192 & 40 & QPSK & & & $67.22,89.62,100.83$ & 8.20 & \\
\hline \multirow{4}{*}{$\begin{array}{c}\text { Double-layer } \\
\text { turbocode }\end{array}$} & 1024 & 40 & 16QAM & \multirow{4}{*}{$\begin{array}{l}16.90, \\
22.53, \\
25.35\end{array}$} & \multirow{4}{*}{$\begin{array}{l}135.11, \\
180.14, \\
202.67\end{array}$} & $130.03,173.37,195.04$ & 63.50 & \multirow{4}{*}{$\begin{array}{c}\text { Low } \\
\text { SNR }>10 \mathrm{~dB}\end{array}$} \\
\hline & 2048 & 40 & 16QAM & & & $132.52,176.69,198.78$ & 32.40 & \\
\hline & 4096 & 40 & 16QAM & & & $133.80,178.40,200.70$ & 16.30 & \\
\hline & 8192 & 40 & 16QAM & & & $134.45,179.27,201.68$ & 8.20 & \\
\hline \multirow{4}{*}{$\begin{array}{l}\text { Triple-layer } \\
\text { turbocode }\end{array}$} & 1024 & 24 & 64QAM & \multirow{4}{*}{$\begin{array}{l}25.30, \\
33.73, \\
37.95\end{array}$} & \multirow{4}{*}{$\begin{array}{l}202.66, \\
270.21, \\
303.99\end{array}$} & $198.02,264.02,297.03$ & 64.50 & \multirow{4}{*}{$\begin{array}{c}\text { Medium } \\
\text { SNR }>16 \mathrm{~dB}\end{array}$} \\
\hline & 2048 & 24 & 64QAM & & & $200.32,267.08,300.47$ & 32.60 & \\
\hline & 4096 & 24 & 64QAM & & & $201.48,268.64,302.22$ & 16.40 & \\
\hline & 8192 & 24 & 64QAM & & & $202.07,269.42,303.11$ & 8.22 & \\
\hline \multirow{4}{*}{$\begin{array}{l}\text { Quadruple-layer } \\
\text { turbocode }\end{array}$} & 1024 & 24 & 256QAM & \multirow{4}{*}{$\begin{array}{c}33.80, \\
45.07, \\
50.70\end{array}$} & \multirow{4}{*}{$\begin{array}{l}270.22, \\
360.28, \\
405.33\end{array}$} & $264.03,352.03,396.04$ & 64.50 & \multirow{4}{*}{$\begin{array}{c}\text { High } \\
\text { SNR }>22 \mathrm{~dB}\end{array}$} \\
\hline & 2048 & 24 & 256QAM & & & $267.09,356.11,400.63$ & 32.60 & \\
\hline & 4096 & 24 & 256QAM & & & $268.64,358.18,402.97$ & 16.40 & \\
\hline & 8192 & 24 & 256QAM & & & $269.43,359.23,404.14$ & 8.22 & \\
\hline
\end{tabular}

Tab. 1. Modem configuration summary.

it is passed for further processing by the higher layers of the modem.

\section{Experimental Measurement}

The transmitter consists of an SD-card memory, a controller, 16-bit DA converter and a wideband Tx amplifier. A signal generated from MATLAB was stored in the SDcard memory. The signal contained 3600 different packets with random data. It was periodically transmitted into the power line at voltage level of 1 Vrms. The Tx amplifier output impedance was $Z_{S}=68 \Omega$ resistive. The received signal went through a 1st-order RC high-pass filter with cut-off frequency of $1.5 \mathrm{kHz}$ to remove the $50 \mathrm{~Hz}$ components. Then it was received using a storage oscilloscope Tektronix DPO4032. The received signal was processed offline in MATLAB. The power line was a $4928 \mathrm{~m}$ long threephase $22 \mathrm{kV}$ overhead line (system with non-grounded neutral point); it is shown in Fig. 10. The line runs from a substation approximately $700 \mathrm{~m}$ in parallel with three other $22 \mathrm{kV}$ power lines and a $3 \mathrm{kV}$ DC railway trolley. Then it crosses the railway and runs separately (see Fig. 10). The power line direction is towards a nearby MW radio transmitter at $620 \mathrm{kHz}$, which induced $1.1 \mathrm{Vrms}$ signal into a $68 \Omega$ load connected between L1 and GND. Two measurement setups were used (see Fig. 11). In the first setup, (Fig. 11b), the measuring signal was injected between phase L1 and GND. GND was connected to the substation's common grounding point; this means that considerable $50 \mathrm{~Hz}$ current from other transformers' neutrals in the substation went through GND. Another anticipated source of GND noise was the railway trolley [23]. On the receiving side in the field, it was grounded using the grounding strip of the disconnector (equipment of the pole). In the second setup (Fig. 11a), the signal was injected between phases L1 and L2. In this setup, the noise level was substantially lower. More information about the line, coupling unit and measurement that helped to design the measurement can be found in [24]-[28].

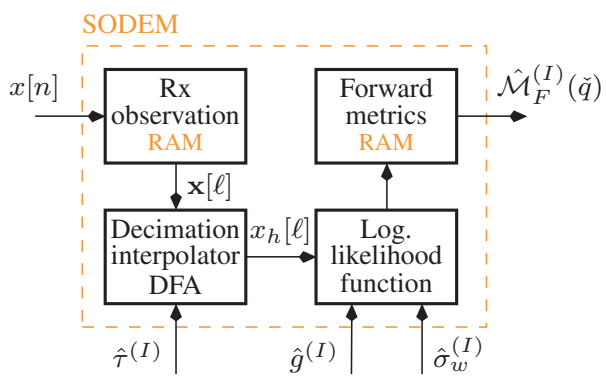

Fig. 8. Block diagram of the soft-output demodulator.

\subsection{Testing Board Description}

The testing board consists of: (1) Input voltage divider, (2) preamplifier with high input impedance, (3) highpass filter with cutoff frequency $(-3 \mathrm{~dB})$ of $1.5 \mathrm{kHz}$, (4) two channel 24-bit sigma-delta ADC with sampling frequency of $187.5 \mathrm{kHz}$, (5) amplitude level indicator, (6) DSP microcontroller with SD card memory, (7) 16-bit sigma-delta DAC with sampling frequency of $187.5 \mathrm{kHz},(8)$ power amplifier capable to deliver 3 Watts and $10 \mathrm{~V}$ peak-peak into the tested power line, with selectable gain (9) optoisolated inputs to obtain time synchronization between receiver and transmitter. The board can be switched between RX (receive) and TX (transmit) operation mode. In the case of TX the board transmits data stored in SD card memory. In the case of $\mathrm{RX}$, the board receives and stores two channels of RX sig- 


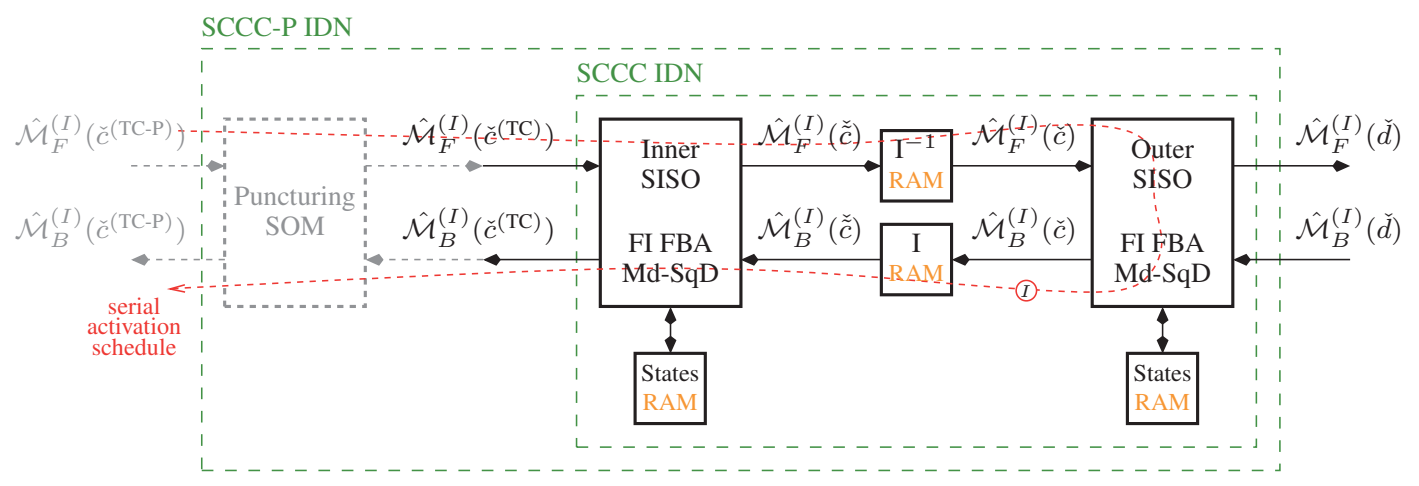

Fig. 9. Block diagram of the iterative detection network.

nal onto SD card memory. First channel is unfiltered (raw) signal from power line, second channel is the same signal filtered by high pass filter to obtain higher dynamic range in frequency band of interest. The amplitude level indicator helps to set input voltage divider to avoid signal clipping and saturation of ADC. For the purposes of analyses in this article, only TX mode was used because of low ADC sampling frequency.

\subsection{Developed Modem Performance Analysis}

Performance analysis of the proposed concept was based on periodic transmission of a testing sequence (modulated signal) into a $22 \mathrm{kV}$ power line from a substation, and subsequent detection of the received signal using a digital storage oscilloscope - see Fig. 10. The modulated MCM signal used 6 subchannels, each of them containing 600 packets composed of 256 symbols. So, 3600 different packets were analyzed during one run. The subchannels (carriers) were centered at frequencies from $30 \mathrm{kHz}$ to $80 \mathrm{kHz}$ with $10 \mathrm{kHz}$ step, and the total MCM signal bandwidth was $84.75 \mathrm{kHz}$. In every subchannel we used linear QPSK modulation with SqRRCS modulation impulse with relative length of 12 symbol periods and rolloff factor of 0.0667. Usable subchannel bandwidth was $9.5 \mathrm{kHz}$ and symbol rate was $8.906 \mathrm{kBd}$. The DAC sampling frequency of $187.5 \mathrm{kHz}$ creates Nyquist zone $93.75 \mathrm{kHz}$ wide, into which the entire MCM modulation could fit with sufficient margin. With respect to the sampling frequency and other parameters listed above, the resulting symbol period was $0.1123 \mathrm{~ms}$, and one symbol period corresponds to 20 samples of the modulated signal.

Serial single-layer $\left(K^{\prime}=1\right)$ turbo code with code rate of $1 / 2$ without puncturing (see Fig. 4) was used as channel coder. Subchannel bitrate was $8.906 \mathrm{kbit} / \mathrm{s}$, so MCM total bitrate was $53.4366 \mathrm{kbit} / \mathrm{s}$. A redundant systematic backward recursive convolution coder having 4 internal states, 8 trellis transitions and code rate of $1 / 2$ was used outer coder. A discrete integrator with a code rate of $1 / 1$ and modulo 4 arithmetic was used as inner coder.

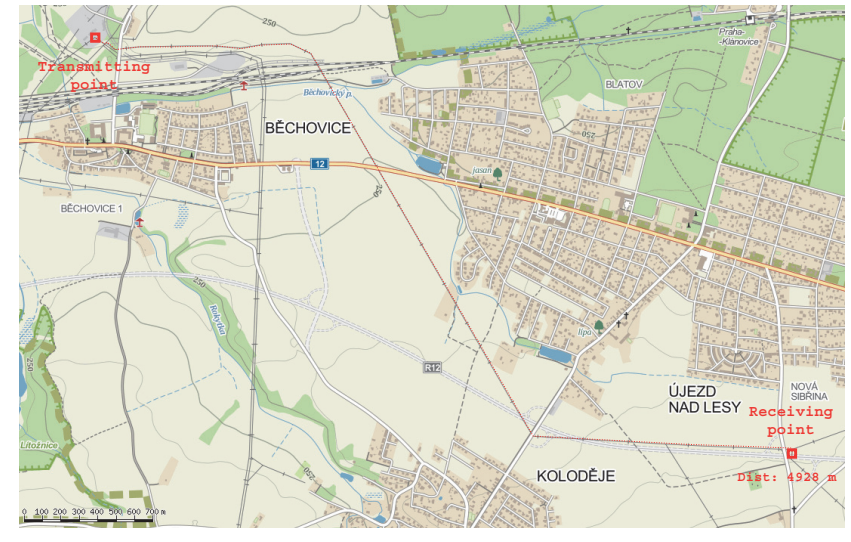

Fig. 10. Power line used for the experimental transmission.

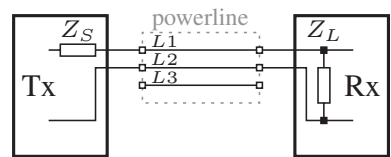

(a) Transmission between two phases.

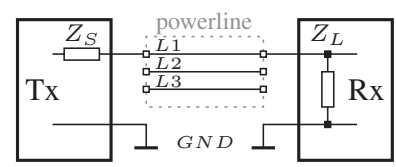
and ground. (b) Transmission between a phase

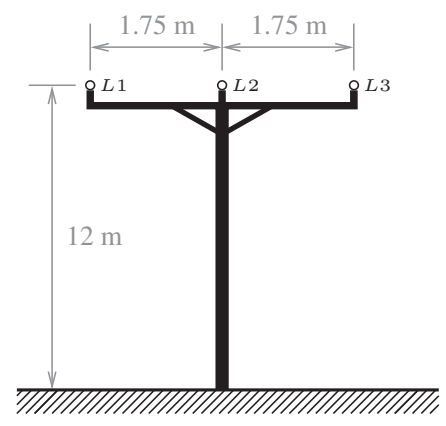

(c) Dimensions of a electric pole of the measured power line.

Fig. 11. Measurement setup. 


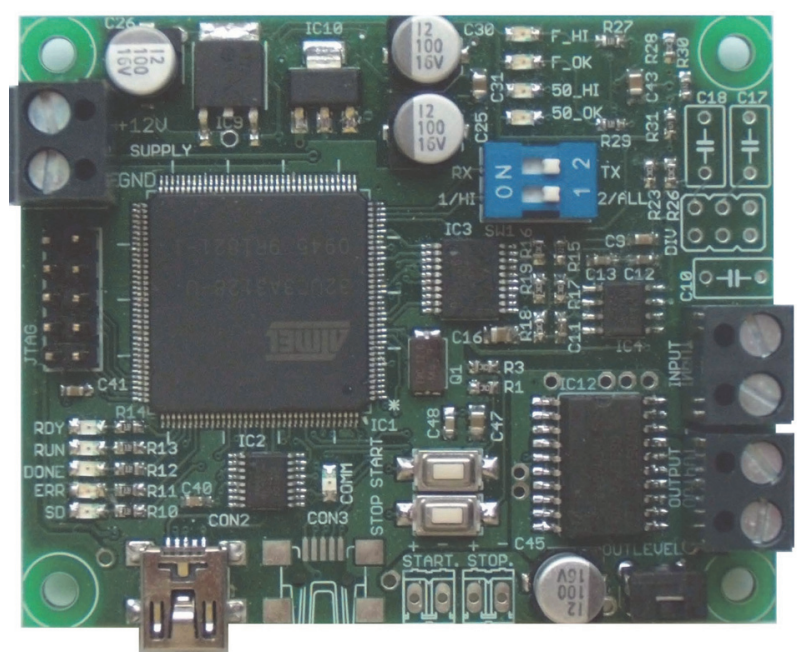

(a) Top view.

Fig. 12. Testing board.
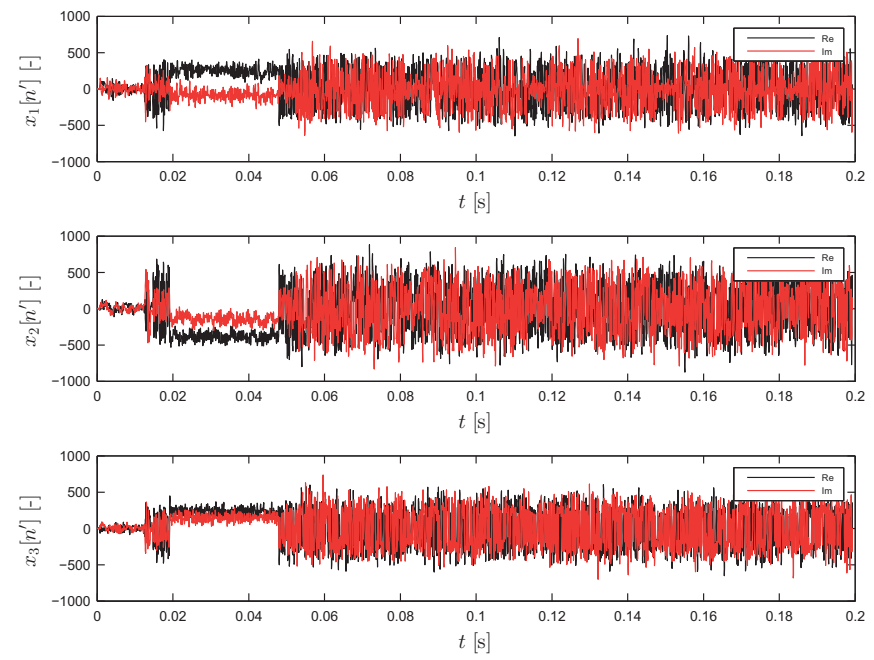

Fig. 13. Complex envelopes of the first 5 received packets.

Serial approach was applied to the packet transmission - see Fig. 1. After subtracting the synchronization overhead (Rudin-Shapiro preambles), the resulting bitrate available for user data is $46.2154 \mathrm{kbit} / \mathrm{s}$. The MCM signal was transmitted repeatedly with idle periods between the repetitions. The beginning of MCM signal in all subchannels was indicated by an empty (null) packet, which helps to easily find the beginning of the received sequence and to extract the segment of the received signal. The complete extracted segment was subsequently analyzed in MATLAB, including determination of error rate through comparing of the received sequence with reference data. The same segment of the testing MCM signal was used for transmission between two phases (Fig. 11a) and between a phase and ground (Fig. 11b). The received signal was always sampled at $250 \mathrm{kHz}$; so, resampling to $187.5 \mathrm{kHz}$ was needed before further processing of the received signal, using a digital interpolator based on Farrow algorithm.

Figure 13 shows complex envelopes of the first 5 packets of the testing sequence at the output of the matched filter,

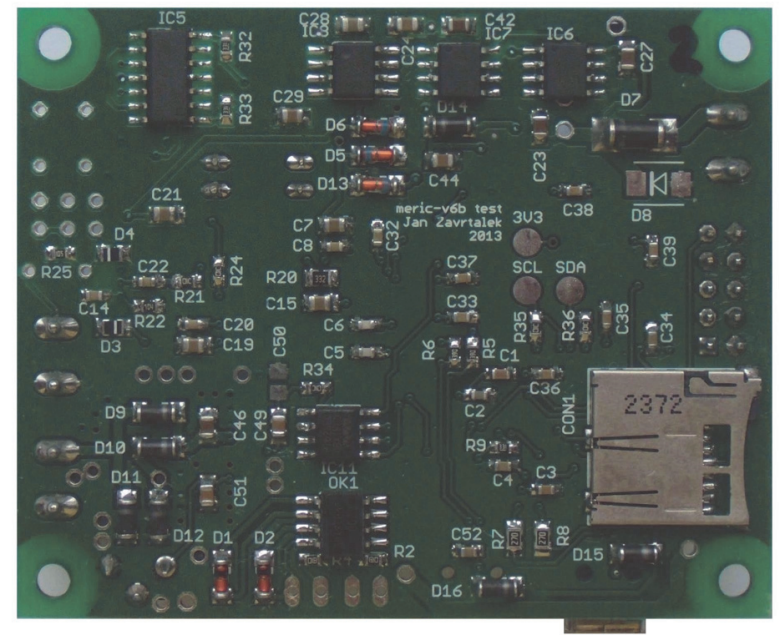

(b) Bottom view.
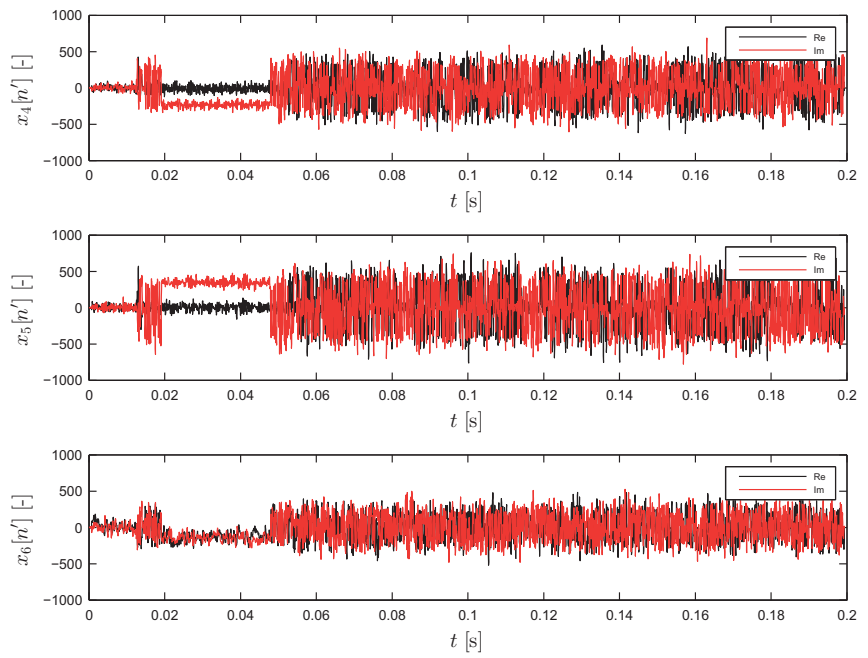

using the measurement setup from Fig. 11a. The number of samples per symbol period of the envelope on the receiving side was set to 4. Graphs in Fig. 13 clearly show the empty (null) leading packets preceded by the synchronization Rudin-Shapiro preambles containing 32 symbols, extended with 8 symbols of cyclic prefix to the total length of 40 symbols. Every packet begins with one of the two RudinShapiro preambles, depending on the service data stream with average bitrate of $0.1805 \mathrm{kbit} / \mathrm{s}$ for the entire MCM bandwidth.

The same complex envelopes are shown also in Fig. 14; Figure 14a shows their spectra, and Fig. 14b constellations. The empty (null) packets are again clearly visible as dense clusters of samples.

Figure 15 shows waveforms of the estimates of channel (subchannel) CSE in the individual frame synchronizers for the first 5 received packets of the testing sequence. The purpose of these dual estimators is to provide synchronization; their function is supported by the data in the form of the $1 \mathrm{st}$ 

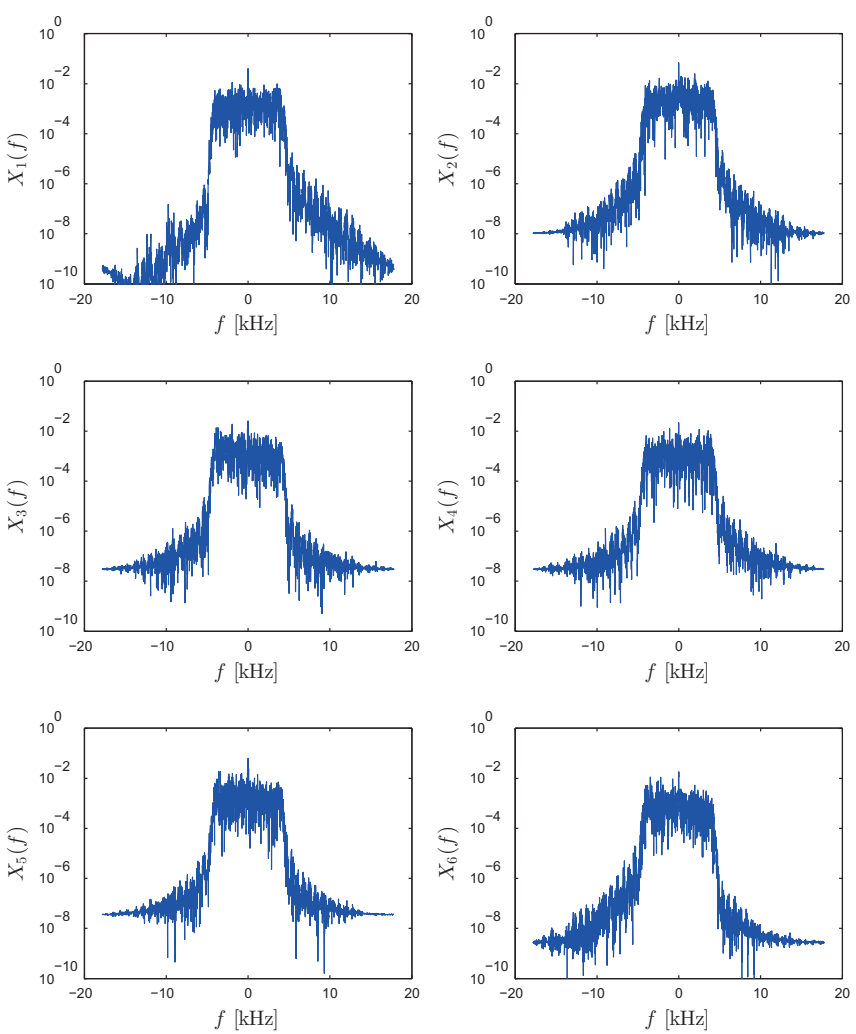

(a) Spectra.

Fig. 14. Spectra and constellations of the first 5 received packets.

or 2nd Rudin-Shapiro preamble. At the output of the internal CSEs there are three basic estimates: initial subchannel symbol timing LAG est. $\equiv \hat{\tau}^{(0)}$, initial subchannel transfer function CSI est. $\equiv \hat{g}^{(0)}$, and initial subchannel signal-to-noise ratio SNR est. $\propto \hat{\sigma}_{w}^{(0)}$ (not shown in Fig. 15).

The outputs of the internal CSEs are immediately used in frame synchronizers to compute logarithmic likelihood functions corresponding to the 1st and 2nd Rudin-Shapiro preamble; Figure 16 shows the values of these functions for the first 5 received packets. The difference between these likelihood functions provides the resulting statistic (LLFR). LLFR is then compared to the threshold given by reduced energy of subchannel complex envelope ( \pm THR.). If LLFR exceeds the value of THR, the beginning of a packet is detected along with the corresponding bit of service data stream.

Now let us leave the zoomed area of the first 5 packets transmitted between two phases, as illustrated in Fig. 11b, and have a look on time lapse graphs of the whole transmission to compare both arrangements. Figure 17 shows the key control statistics of the receiver. First of all, every transmitted packet contains its 7-bit identification number (ID) that is used for packet loss analysis. Peak triplet flag indicates time spacing of synchronization peaks at the output of frame synchronizer; this flag shows whether both synchronization peaks of the previous and of the next packet occur in correct time interval - therefore the evaluation of the Peak triplet flag is delayed by one packet. If the synchronization peaks cannot be localized, the flag is set to zero, which means (false)
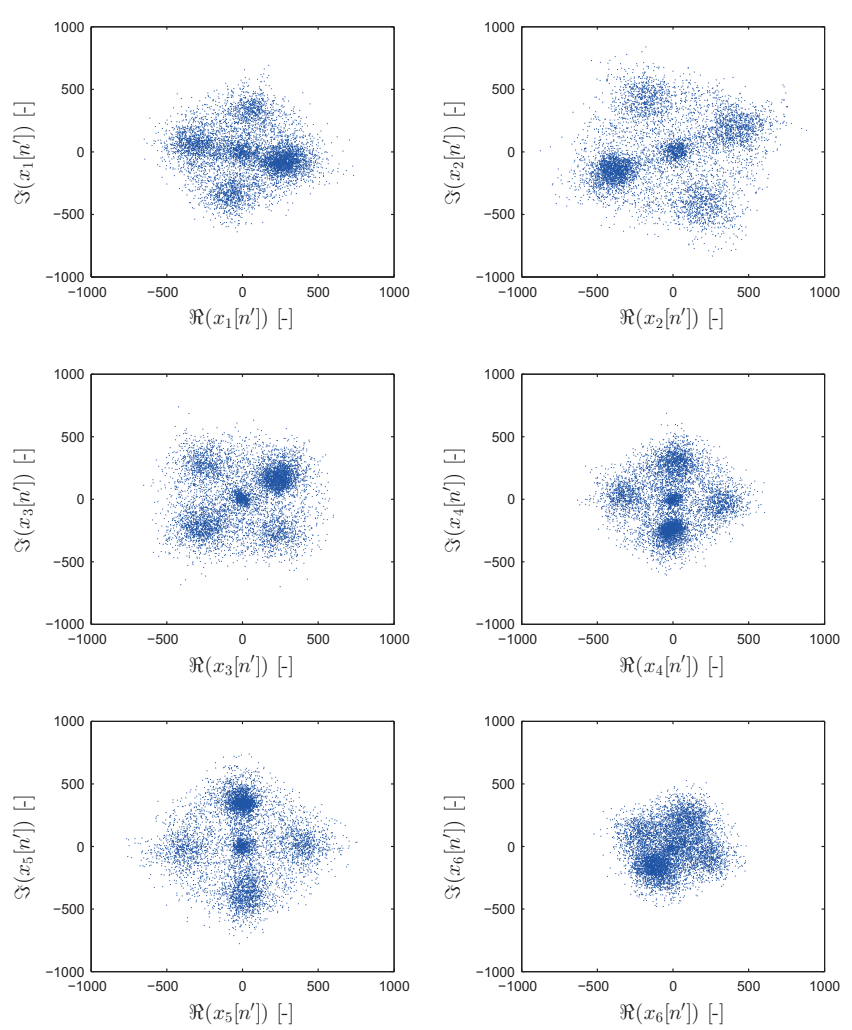

(b) Constellations.

frame synchronization alarm or another error on the receiving side. These two statistics together influence the value of packet Validity flag; a packet is considered to be valid if the extracted ID fits into the assumed sawtooth sequence and the Peak triplet flag is not set to zero.

Figure 18 shows symbol error rates (SER) of the received packets. It is visible that the transmission between a phase and ground (Fig. 18b) suffered from higher noise levels, and therefore the symbol error ratio is higher than for transmission between two phases (Fig. 18a). In both cases we can see decreased SER after repeated iterations of SODEM in Fig. 7. The most attenuated and most noisy subchannel in both cases was the subchannel number 6. All the other channels were decoded with almost no errors during the whole transmission. Rarely there appear packets with very high symbol error rate, which almost leads to their loss; these situations cause singular faults of symbol timing synchronization in receiver tracking mode, i.e. inside the SODEM loop in Fig. 7. The speed of convergence towards correct data estimates during SODEM iterations depends also on packet length. In this experiment we used relatively short packets of 256 symbols, due to time constraint. For practical use it is necessary to transmit packets with minimum length of 1024 symbols in order to increase the turbo code performance.

Figure 19 shows histograms of channel parameters estimates (for a $22 \mathrm{kV}$ overhead line) from synchronization acquisition mode. These estimates are obtained from the in- 

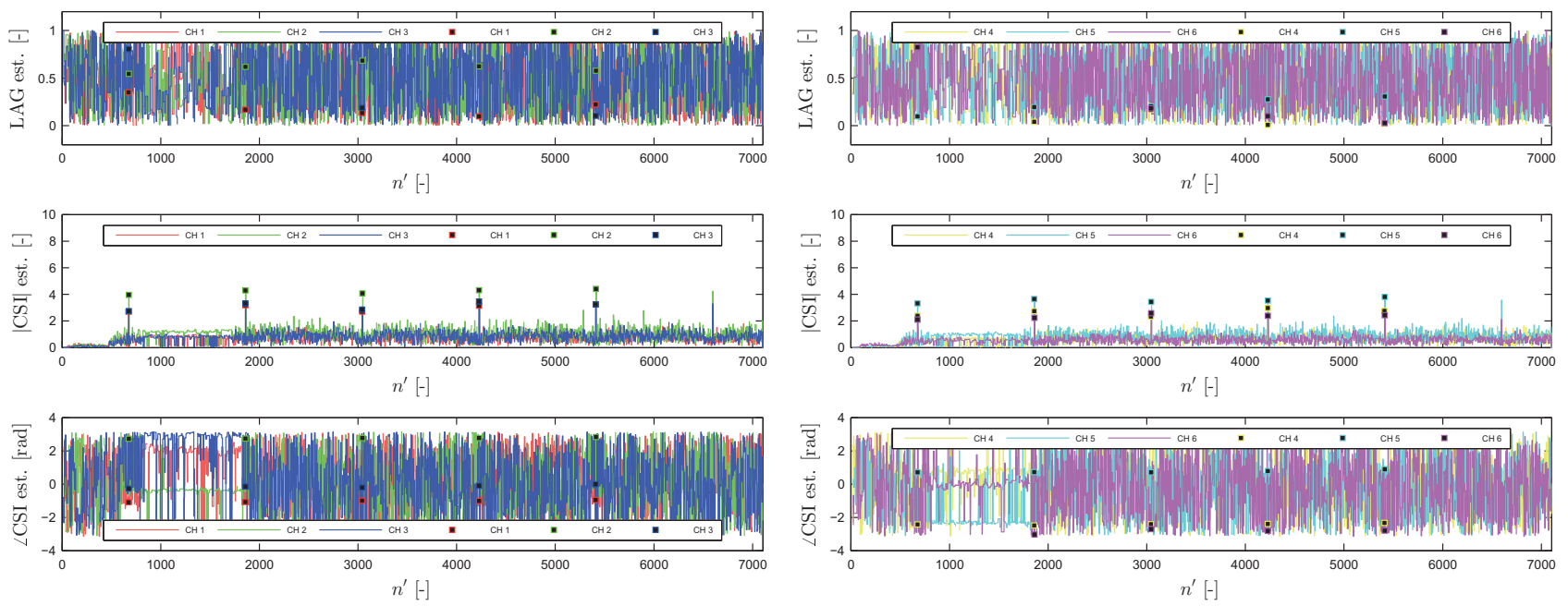

Fig. 15. Parameter estimates for the first 5 received packets (synchronization acquisition mode).
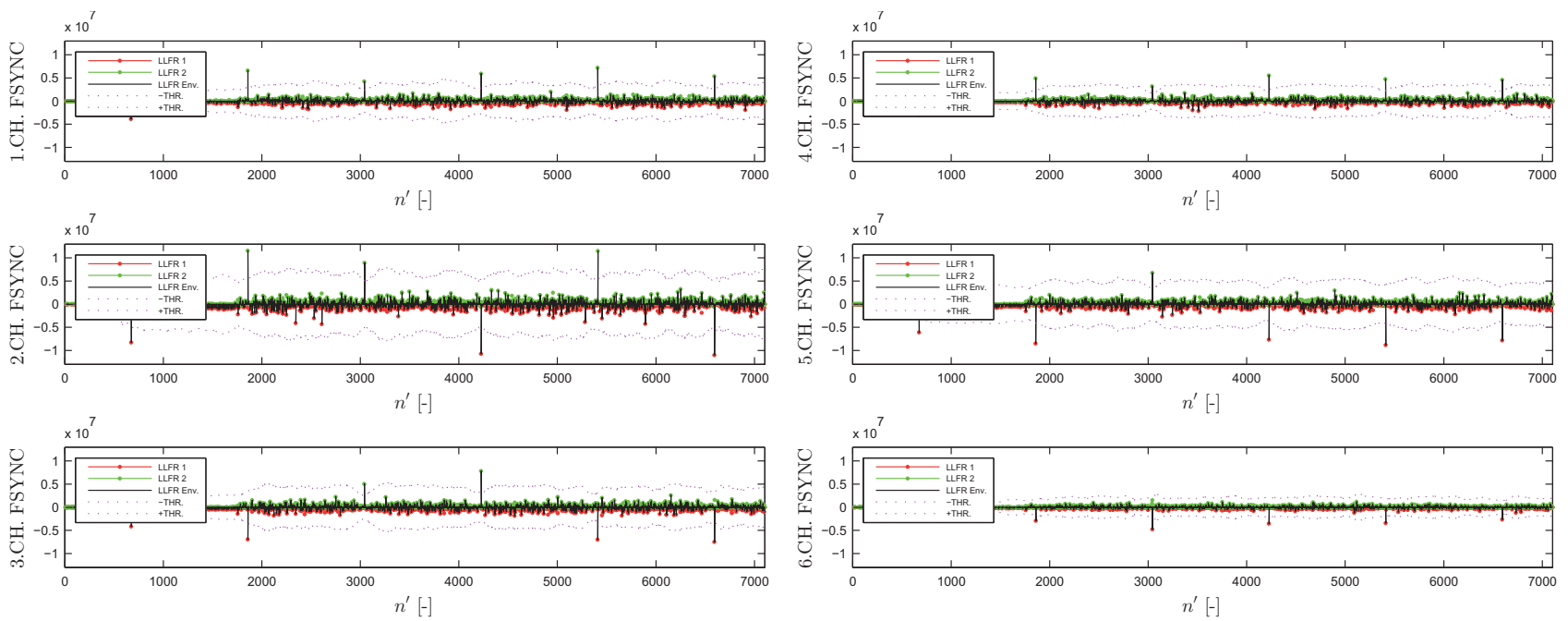

Fig. 16. Sync. stats for the first 5 received packets.

ternal CSEs in the individual frame synchronizers. It is evident that relatively low values of SNR were achieved for both arrangements. Higher values of SNR (and thus better communication conditions) were observed for the arrangement with transmission between two phases (Fig. 11a). The average SNR values were between $0 \mathrm{~dB}$ and $8 \mathrm{~dB}$, most often $4 \mathrm{~dB}$. For the arrangement with transmission between a phase and ground (Fig. 11b), the situation was even worse - the average SNR values were between $-2 \mathrm{~dB}$ and $7 \mathrm{~dB}$, most often only $1 \mathrm{~dB}$. The SNR in this configuration allows only transmission with QPSK constellation at the best, but not higher-order ones. From $|\mathrm{CSI}|$ histogram in Fig. 19 we can see that the most attenuated subchannel was the 6th one. In both arrangements, the SNR of subchannel number 2 was the best. Phase estimates $\angle \mathrm{CSI}$ est and symbol timing LAG est. had uniform distribution for all channels, which corresponds to the expectations.

Figure 20 shows the estimates LAG est., CSI est. and SNR est. from frame synchronizers shown in time lapse for the whole transmission. Attention should be paid to the sawtooth-shaped estimate of symbol timing LAG est; it indicates that receiver ADC sampling frequency (after resampling) was lower than transmitter DAC sampling frequency. This difference also causes slow rotation of phase estimate $\angle$ CSI est., and thus slow rotation of the QPSK constellation.

Figure 21 shows the estimates from receiver tracking mode $\left(\right.$ LAG est. $\equiv \hat{\tau}^{(I)}$, CSI est. $\equiv \hat{g}^{(I)}$ and SNR est. $\left.\propto \hat{\sigma}_{w}^{(I)}\right)$ measured at the output of the SODEM switch for the 1st and the 3 rd system iteration $I$. The time scale is the same as in Fig. 20. After comparing the graphs in Fig. 20 and Fig. 21 we can conclude that repeated iteration yields more accurate estimates. This means that SODEM loop in Fig. 7 converges, i.e. it works in accordance with theoretical assumptions.

Figure 22 illustrates the average amplitude spectra i.e., spectrum of the transmitted MCM signal and spectra of the received MCM signals for both arrangements.

Finally, Figure 23 shows channel transfer characteris- 

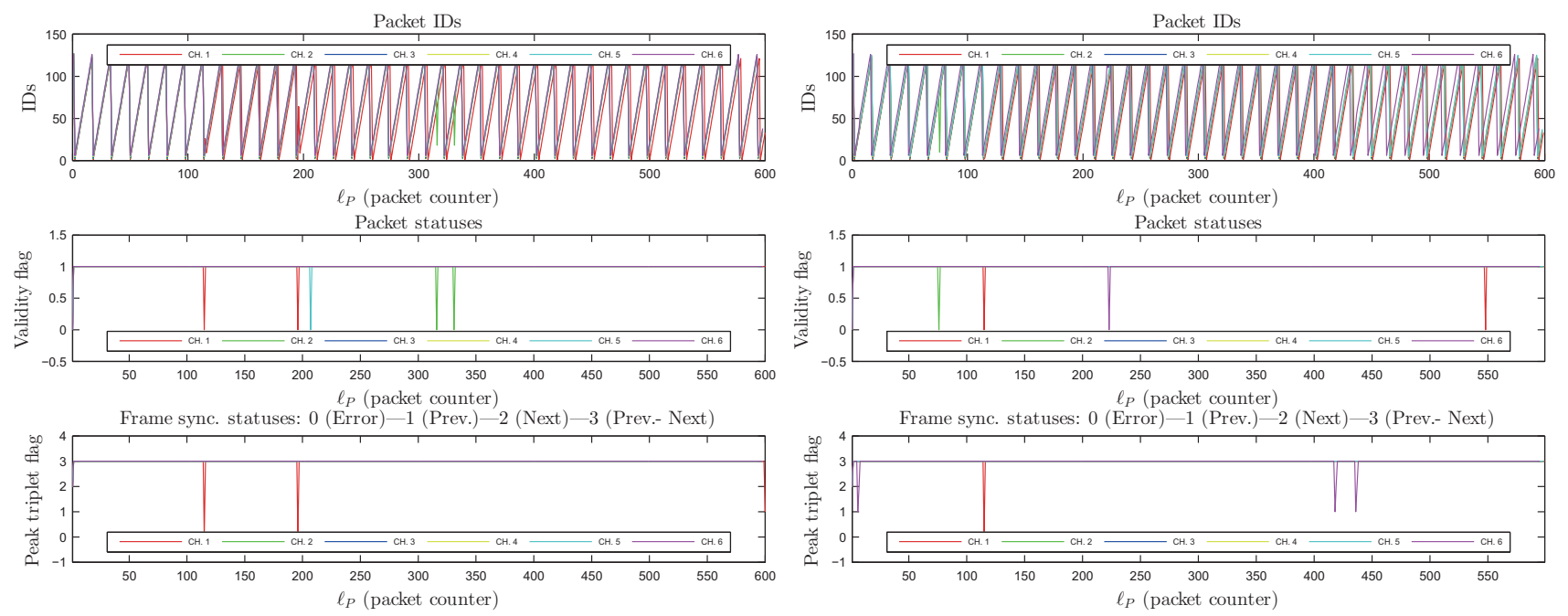

(a) Transmission between two phases.

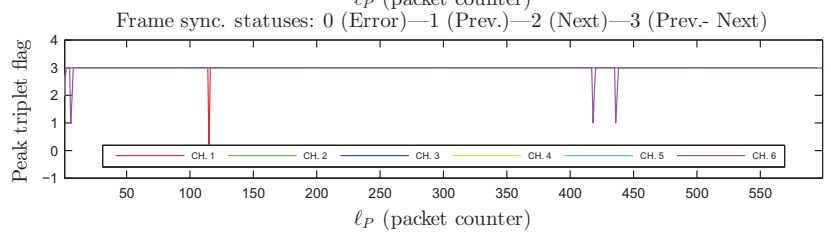

(b) Transmission between a phase and ground.

Fig. 17. Packet IDs and flags (Peak triplet flag, Validity flag).
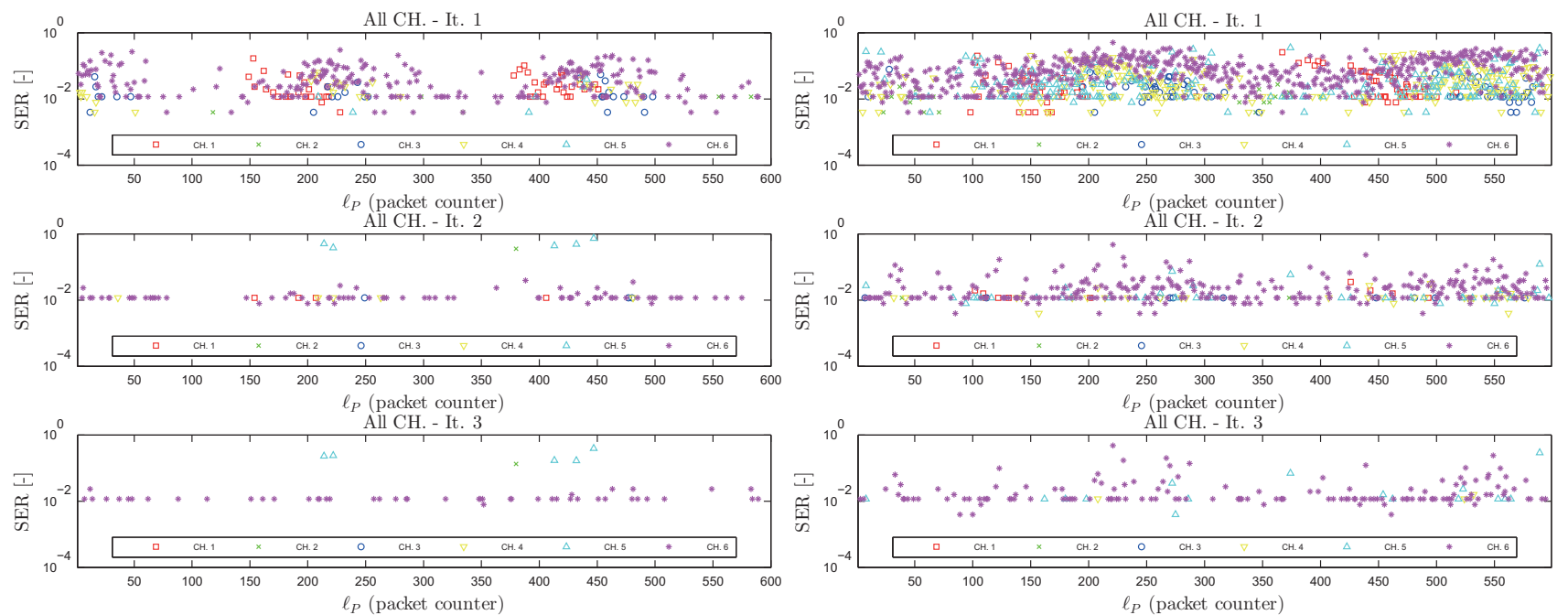

(a) Transmission between two phases.

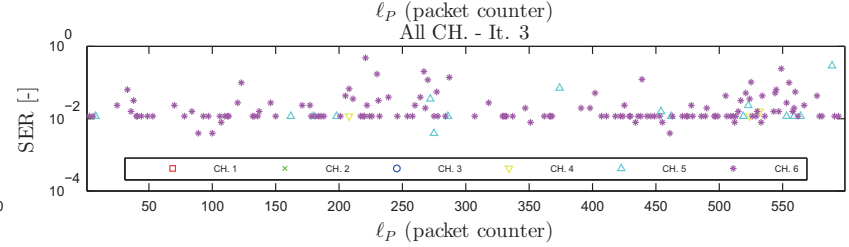

(b) Transmission between a phase and ground.

Fig. 18. Symbol error ratios.

tics of the $22 \mathrm{kV}$ line resulting from interpolation of the isolated $|\mathrm{CSI}|$ estimates for all subchannels within the frequency band in fixed time. These characteristics are shown for estimates of attenuation obtained in synchronization acquisition mode, as well as for estimates in receiver tracking mode (after the 3rd system iteration). It is noticeable that the channel is stationary in time, with moderate frequency selectivity.

\section{Conclusions and Future Work}

The proposed concept has two major drawbacks: it introduces high latency (because of small bandwidth of particular subchannels, and thus low bitrate of the individual disjoint streams), and also high requirements on memory of the layered turbo decoder. On the other hand, this solution allows (in collaboration with higher layers of the receiver) adaptive setting of subchannel power level as well as adap- tive subchannel modulation and coding. In extreme case, only one subchannel is needed for communication (then MCM modulation becomes single-carrier in the most suitable frequency band). This should be the subject of future research.

These drawbacks can be eliminated by using MCM with parallel transmission. Such communication uses the whole available MCM bandwidth at once. All subchannels are then used together for communication in a single, fast data stream. The individual packets are situated in timefrequency domain as shown in Fig. 24. When we compare this approach with serial transmission (Fig. 1), it is clear that latency decreases $\mathrm{K}$ times. When some of the subchannels is heavily disturbed or deformed by a gap in the frequency characteristics of the given channel, the interleaver in the turbodecoder spreads the block errors into isolated errors which are eliminated in the iterative detection process. We expect that this approach can work up to $25 \%$ of lost subchannels at 

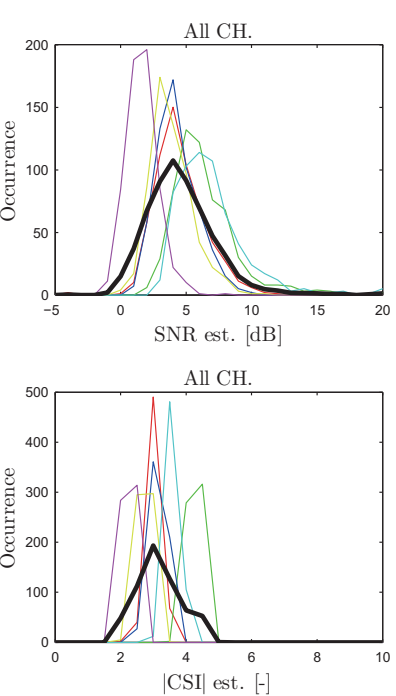

(a) Transmission between two phases.

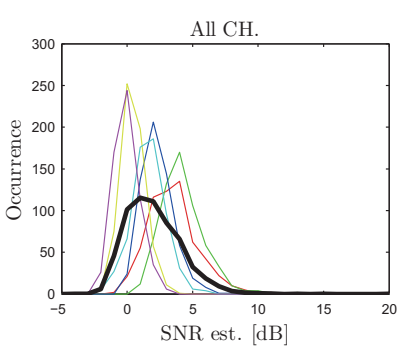

All CH.

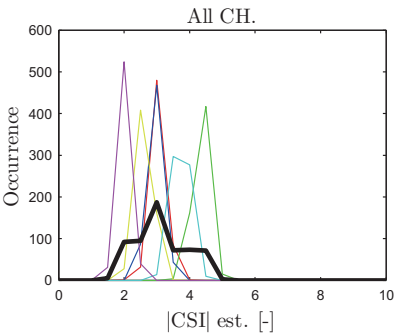

(b) Transmission between a phase and ground.
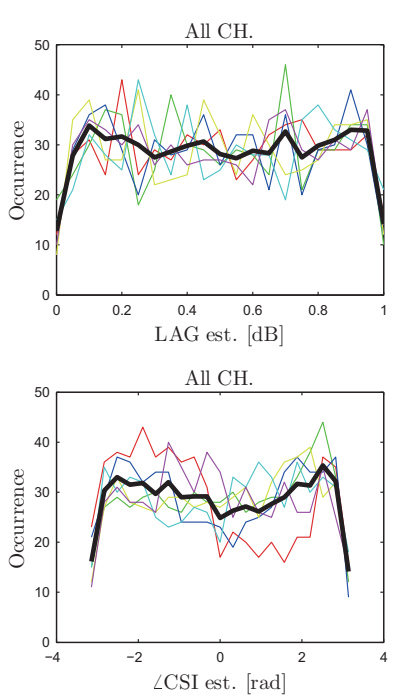

Fig. 19. Histograms of parameter estimates (synchronization acquisition mode).
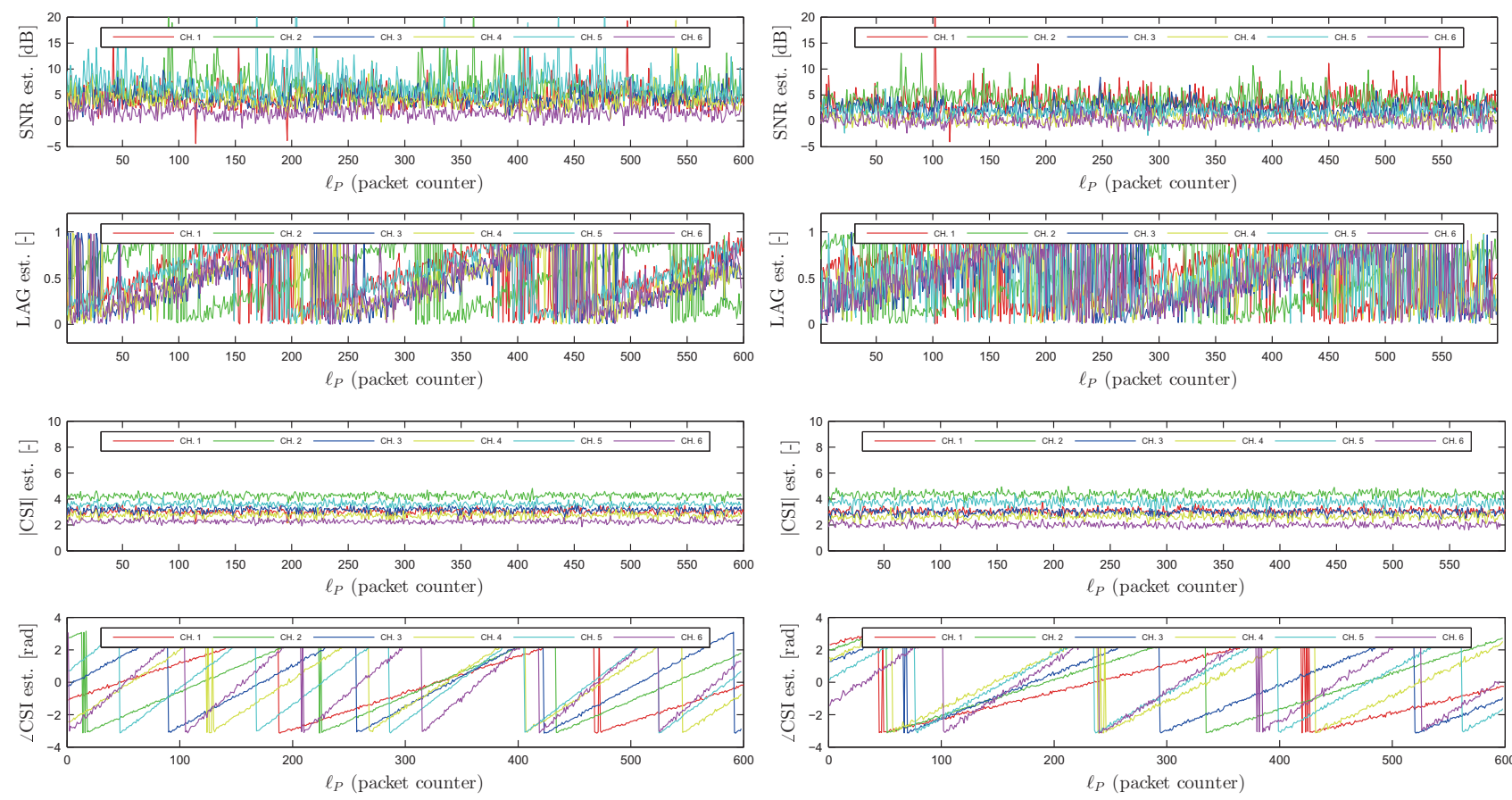

(a) Transmission between two phases.

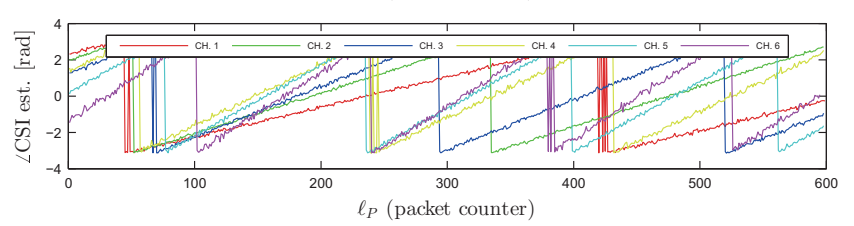

(b) Transmission between a phase and ground.

Fig. 20. Parameters estimates (synchronization acquisition mode).

turbo coder rate of $1 / 2$. Adaptive subchannel modulation and coding is also possible, but it puts high demands on management in higher layers.

In the parallel modem configuration with reduced latency, the transmitter scheme will be very similar to the current design Fig. 2. except the turbo coder setup. The set of turbo coder blocks working low bitrates will be replaced by one, $K$-times faster turbo coder. The single output sequence $q$ will then be segmented in serial-parallel converter into $K$ segments with equal lengths that will be stored separately in a memory. The converter will be equipped with 2

such memory blocks working alternately. When the first block is being filled with new data, the previously stored data will be read from the second block and passed to modulators. Then the roles of the blocks will be switched.

Similarly to the serial configuration, both punctured turbo coding (Fig. 4) and hierarchical QAM mapping can be used. The mapping block must be slightly modified by adding shift registers to receive sequential data. Depending on constellation, the turbo coder can work at $2 K$ rate (for 16QAM without puncturing), $3 K$ rate (64QAM without puncturing), or $4 K$ rate (256QAM without puncturing). 

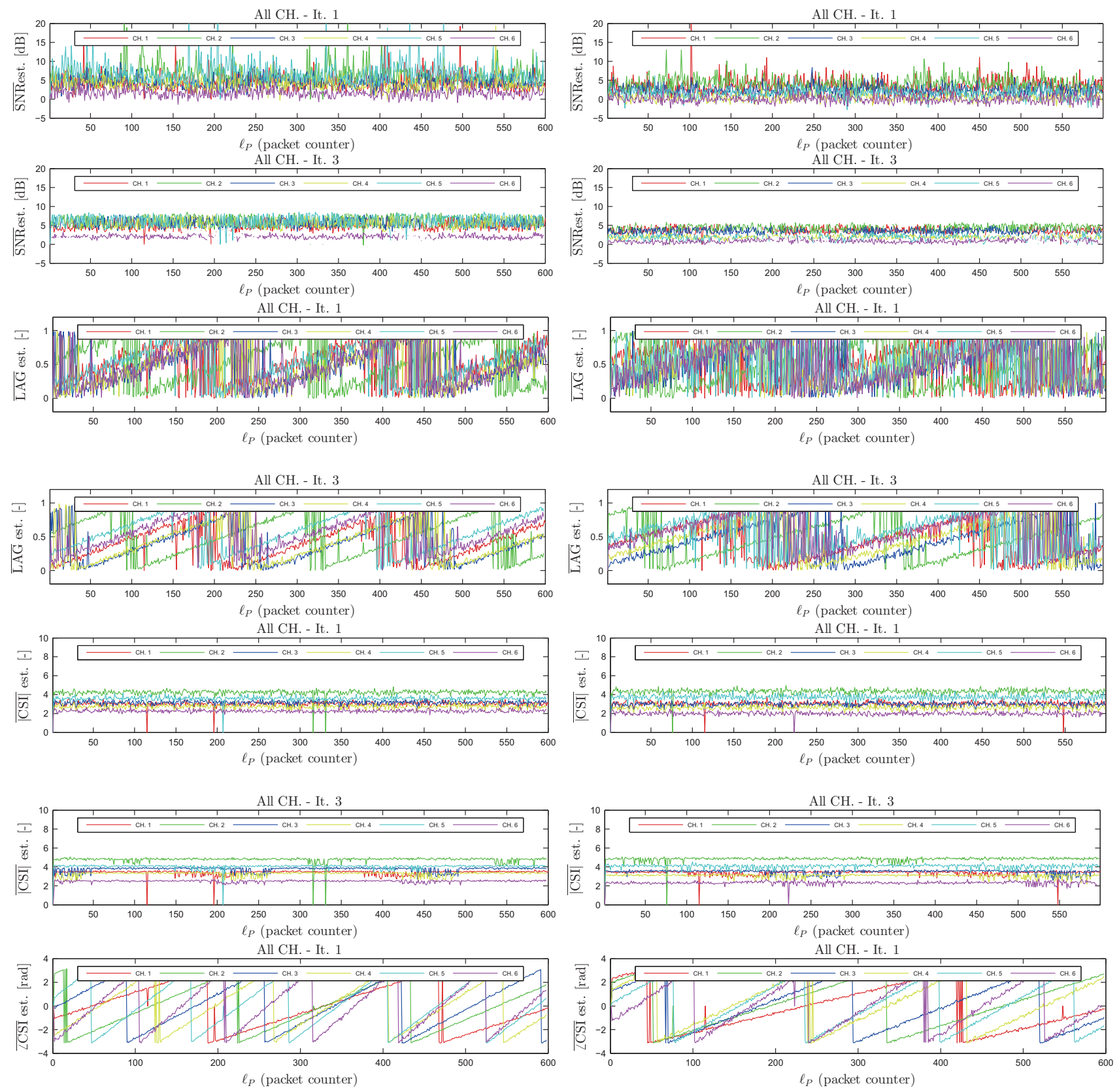

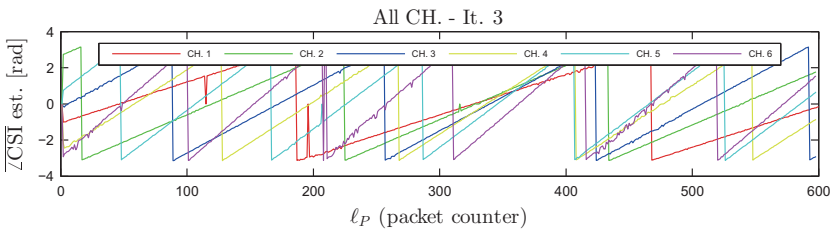

(a) Transmission between two phases.

Fig. 21. Parameters estimates (tracking mode synchronization).

The receiver side of the modem with parallel MCM will be also very similar to the current design (Fig. 6). The main difference consists in fully synchronous reception and processing of all subchannels. Therefore, the frame synchronizers will be encapsulated into a mutually synchronized bank of frame synchronizers that search for full ( $K$ partial synchronization pulses at once) or partial ( $K^{\prime}<K$ partial syn-

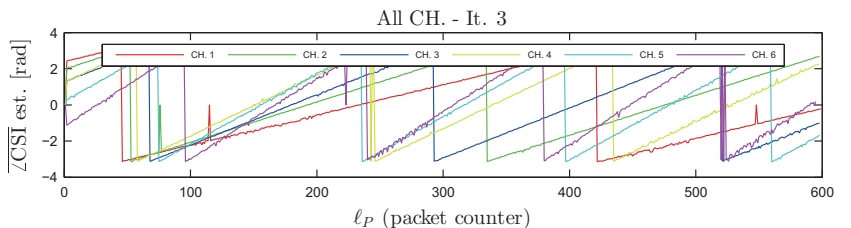

(b) Transmission between a phase and ground.

chronization pulses at once) multidimensional synchronization pulse, depending on the receiver sensitivity setting.

All sections of the complex envelopes, isolated by multidimensional frame synchronizer and corresponding to the respective segments of one data packet, will be passed through the switch into the joint iterative synchronizerdetector. There the processing begins again in soft demodu- 


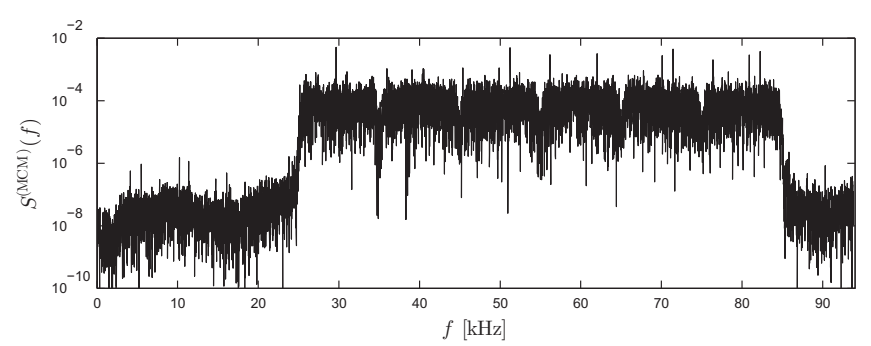

(a) Transmitted signal.

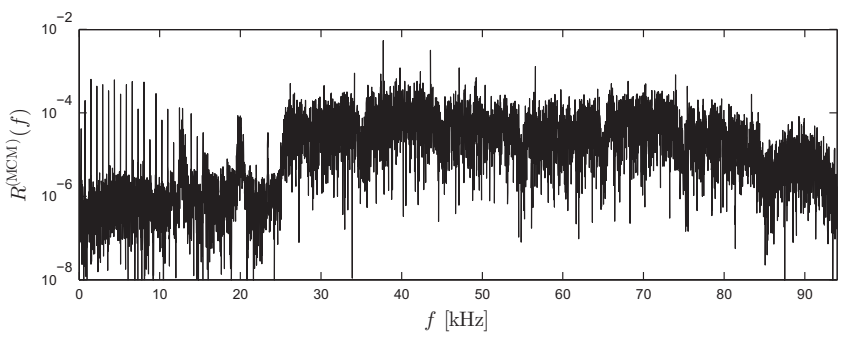

(b) Transmission between two phases.

Fig. 22. Transmitted and received signal spectra.
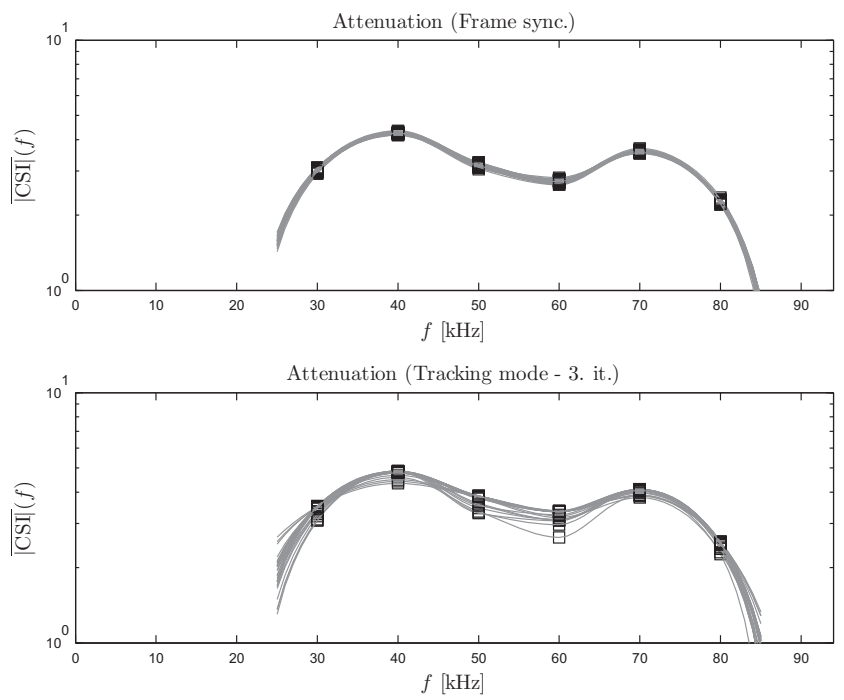

(a) Transmission between two phases

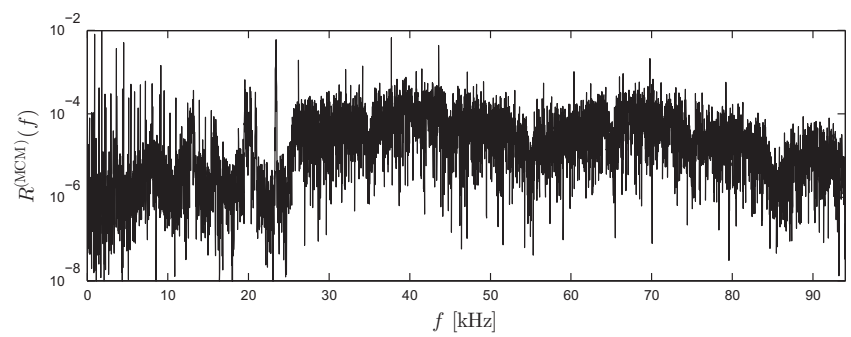

(c) Transmission between a phase and ground.
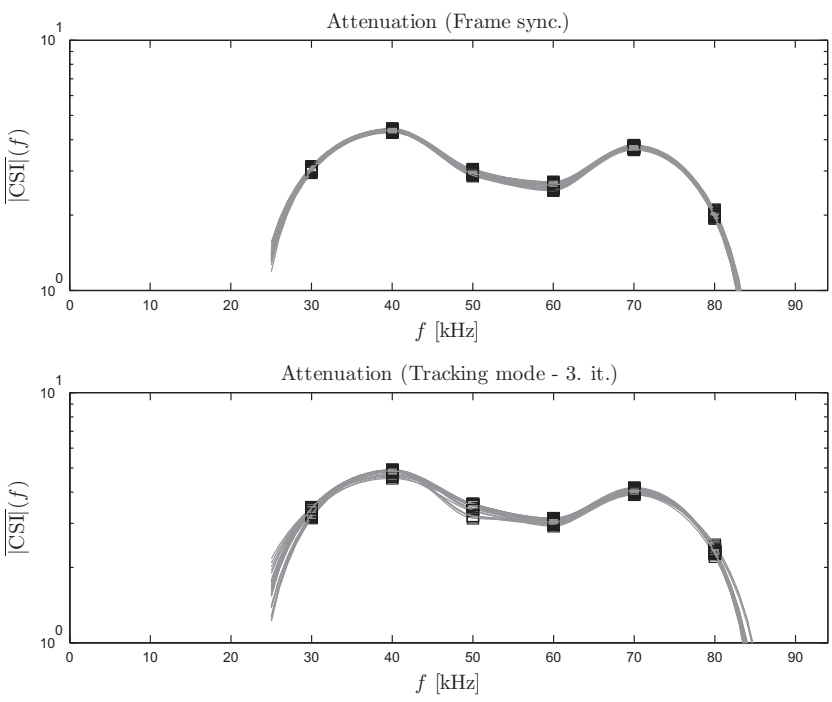

(b) Transmission between a phase and ground.

Fig. 23. Channel transfer function estimate (initial and after the final iteration).

lator, which is now multidimensional and produces the forward metrics corresponding to all particular data segments at once. These metrics are then merged in serial-parallel converter to create a single output stream $\hat{\mathcal{M}}_{F}^{(I)}(\breve{q})$, which is passed into one fast SCCC IDN (Fig. 9). The forward output metric $\hat{\mathcal{M}}_{F}^{(I)}(\breve{d})$ can be thresholded again into hard estimates $\hat{d}$, and the backward output metric $\hat{\mathcal{M}}_{B}^{(I)}(\check{q})$ will be combined with forward metrics $\hat{\mathcal{M}}_{F}^{(I)}(\check{q})$ to form a sequence of posterior probabilities $\hat{\mathcal{P}}^{(I)}(\check{q})$. The sequence will be used to support the estimator of the receiver tracking mode working on a fixed segment (SDD FS CSE). This estimator is similar to SDD EM CSE in synchronizer-detector (Fig. 7); the difference is that the segment length in this case is $L_{S}$, which is identical with the length of packet segments $L / K$. So, during the processing of the complete signal, the CSE is $K$-times reset and the computation of the estimates (initial subchannel attenuation $\left|\hat{g}_{k}^{(0)}\right|$, phase rotation $\angle \hat{g}_{k}^{(0)}$, symbol timing $\hat{\tau}_{k}^{(0)}$ and standard error of AWGN $\hat{\sigma}_{w_{k}}^{(0)}$ ) starts from the beginning. The output of FS CSE will be a set of isolated triplets $\left\{\hat{g}^{(I)}\left[\ell_{S}\right], \hat{\tau}^{(I)}\left[\ell_{S}\right], \hat{\sigma}_{w}^{(I)}\left[\ell_{S}\right]\right\}_{\ell_{S}=1}^{K}$, the elements of which will then be composed together into updated vector estimates $\hat{g}^{(I)}, \hat{\tau}^{(I)}$ and $\hat{\sigma}_{w}^{(I)}$ through the serial-parallel converter. These vectors will be passed back to the input of the multidimensional SODEM, and this operation will conclude one iteration of the synchronizer-detector. We are going to implement and test this solution in the future. 


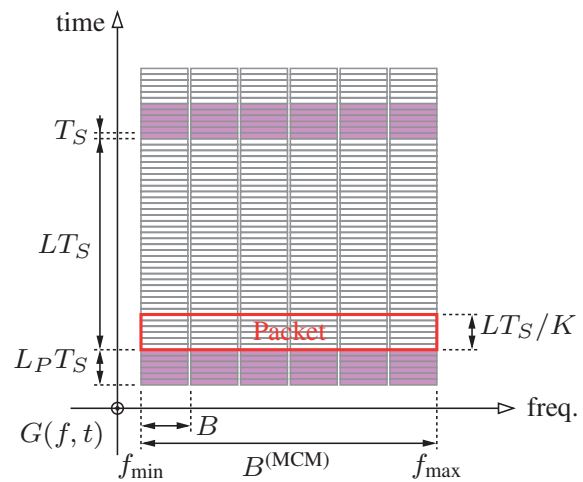

Fig. 24. Time-frequency partition of packets for parallel MCM.

\section{Acknowledgments}

This work was conducted within the project TA03011192 "Research and Development of Newgeneration Communication Devices for Transmission Over High-voltage Power Lines" granted by the Technology Agency of the Czech Republic and it was partially supported by PREdistribuce, a.s. The authors want to thank to Ladislav Musil, Pavel Petrovský, Petr Denemark and Radek Hanuš for their valuable help.

\section{References}

[1] CHUGG, K., ANASTASOPOUlOS, A., CHEN, X. Iterative detection: Adaptivity, Complexity Reduction and Applications. Kluwer Academic Publishers, 2001. ISBN 0-729-37277-8. DOI: 10.1007/978-1-4615-1699-6.

[2] VUCETIC, B., JUAN, J. Space-Time Coding. Kluwer Academic Publishers, 2003. ISBN 0-470-84757-3. DOI: 10.1002/047001413X.

[3] VUCETIC, B., JUAN, J. Turbo Codes: Principles and Applications. Kluwer Academic Publishers, 2000. ISBN 0-792-37868-7. DOI: $10.1007 / 978-1-4615-4469-2$.

[4] BERROU, C., GLAVIEUX, A., THITMAJSHIMA, P. Near Shannon limit error correcting coding and decoding: Turbo-codes. In Proceedings of the International Conference on Communications. Geneva (Switzerland), 1993, p. 1064-1070. DOI: 10.1109/ICC.1993.397441

[5] BAHL, L. R., COCKE., J., JELINEK, F., RAVIV, J. Optimal decoding of linear codes for minimizing symbol error rate. IEEE Transactions on Information Theory, 1974, vol.20, no. 2, p. 284-287. DOI: 10.1109/TIT.1974.1055186.

[6] HAGENAUER, J., HOEHER, P. A Viterbi algorithm with softdecision outputs and its applications. In Proceedings of IEEE Global Telecommunications Conference. Dallas (USA), 1989, p. 16801686. DOI: 10.1109/GLOCOM.1989.64230

[7] FORNEY, J. D. The Viterbi algorithm. Proceedings of the IEEE, 1973, vol. 61, no. 3, p. 268-278. DOI: 10.1109/PROC.1973.9030.

[8] GUNTHER, J. H., KELLER, D., MOON, T. K. A generalized BCJR algorithm and its use in turbo synchronization. In Proceedings of Acoustics, Speech, and Signal Processing (ICASSP '05). 2005, p. 837-840. DOI: 10.1109/ICASSP.2005.1415840.
[9] VALLES, E. L., WESEL, R. D, VILLASENOR, J. D., JONES, C. R. Carrier and timing synchronization of BPSK via LDPC code feedback. In Signals, Systems and Computers (ACSSC '06). 2006, p. 2177-2181. DOI: 10.1109/ACSSC.2006.355154.

[10] GINI, F., GIANNAKIS, G. B. Frequency offset and symbol timing recovery in flat-fading channels: a cyclostationary approach. IEEE Transactions on Communications, 2002, vol. 46, no. 3, p. 400-411. DOI: $10.1109 / 26.662646$.

[11] CASADEI, M., CIONI, S., CORAZZA, G. E. Advanced iterative symbol timing recovery for mobile DVB-RCS. In 65th IEEE Vehicular Technology Conference. Dublin (Ireland), 2007, p. 1425-1429. DOI: 10.1109/VETECS.2007.298.

[12] KEKRT, D., LUKES, T., KLIMA, M., FLIEGEL, K. 2D Iterative MAP detection: principles and applications in image restoration. $R a$ dioengineering, 2014, vol. 23, no. 2, p. 618-631.

[13] VARAHRAM, P., ALI, B. M. A low complexity partial transmit sequence for peak to average power ratio reduction in OFDM systems. Radioengineering, 2011, vol. 20, no. 3, p. 677-682.

[14] HERZET, C., RAMON, V., VANDENDORPE, L., MOENECLAEY, M. EM algorithm-based timing synchronization in turbo receivers. In Proceedings of IEEE Conference on Acoustic, Speech, and Signal Processing (ICASSP '03). 2003, vol. 4, p. 612-615. DOI: 10.1109/ICASSP.2003.1202717.

[15] GUENACH, M., WYMEERSCH, H., MOENECLAY, M. Joint estimation of path delay and complex gain for coded systems using the EM algorithm. In 2004 International Zurich Seminar on Communications. Zurich (Switzerland), 2004, p. 216-219. DOI: 10.1109/IZS.2004.1287428.

[16] RAMON, V., HERZET, C., VANDENDORPE, L., MOENECLAEY, M. EM algorithm-based multiuser synchronization in turbo receivers. In Proceedings of IEEE Conference on Acoustic, Speech, and Signal Processing (ICASSP '04). 2004, vol. 4, p. 849-852. DOI: 10.1109/ICASSP.2004.1326960.

[17] SYKORA, J., BURR, A. G. Iterative decoding networks with iteratively data eliminating SDD and EM based channel state estimator. In 15th IEEE Symposium on Personal, Indoor and Mobile Radio Communications (PIMRC '04). 2004, vol. 2, p. 785-790. DOI: 10.1109/PIMRC.2004.1373807.

[18] SYKORA, J., VCELAK, J. Iterative EM based IMD synchronization for fast time-variant channel with subspace order recursive LS iterator. In Asia-Pacific Conference on Communications, 2005, p. 921-925. DOI: 10.1109/APCC.2005.1554197

[19] ANASTASOPOUlOS, A., CHUGG, K. M. Adaptive soft-input soft-output algorithms for iterative detection with parametric uncertainty. IEEE Transaction on Communications, 2000, vol. 48, no. 10, p. 1638-1649. DOI: $10.1109 / 26.871389$

[20] NOELS, N., HERZET, C., DEJONGHE, A., LOTTICI, V., STEENDAM, H., MOENECLAEY, M., LUISE, M., VANDERDORPE, L. Turbo synchronization: an EM algorithm interpretation. In IEEE International Conference on Communications. 2003, vol. 4, p. 2933-2937. DOI: 10.1109/ICC.2003.1204575

[21] HERZET, C., WYMEERSCH, H., MOENECLAY, M., VANDERDORPE, L. On maximum-likelihood timing synchronization. IEEE Transactions on Communications, 2007, vol. 55, no. 6 , p. 1116-1119. DOI: 10.1109/TCOMM.2007.898863

[22] HERZET, C., NOELS, N., LOTTICI, V., WYMEERSCH, H., LUISE, M., MOENECLAY, M., VANDERDORPE, L. Code-aided turbo synchronization. Proceedings of the IEEE, 2007, vol. 95, no. 6, p. 1255-1271. DOI: 10.1109/JPROC.2007.896518 
[23] CARSON, J. R. Wave propagation in overhead wires with ground return. The Bell System Technical Journal, 1926, vol. 5, no. 4, p. 539-554. DOI: 10.1002/j.1538-7305.1926.tb00122.x

[24] ZIMMERMANN, M., DOSTERT, K. A multipath model for the power line channel. IEEE Transactions on Communications, 2002, vol. 50, no. 4, p. 553-559. DOI: 10.1109/26.996069

[25] HOCH, M. Comparison of PLC G3 and PRIME. IEEE International Symposium on Power Line Communications and Its Applications. Udine (Italy), 2011, p. 165-169. DOI: 10.1109/ISPLC.2011.5764384

[26] ALI, S. S., BHATTACHARYA, A., PODDAR, D. R. Design of bidirectional coupling circuit for broadband power-line communications. Journal of Electromagnetic Analysis and Applications, 2012, vol. 4, no. 4 , p. 162-166. DOI: $10.4236 /$ jemaa.2012.44021

[27] GUO, Y., XIE, Z., WANG, Y. A model for $10 \mathrm{kV}$ overhead power line communication channel. In Proceedings of the Second Symposium International Computer Science and Computational Technology (ISCSCT '09). Huangshan (China), 2009, p. 289-292.

[28] NASSAR, M., LIN, J., MORTAZAVI, Y., DABAK, A., KIM, H. I., EVANS, B. L. Local utility powerline communications in the 3-500 $\mathrm{kHz}$ band: channel impairments, noise, and standards. IEEE Signal Processing Magazine, 2012, vol. 29, no. 5, p. 116-127. DOI: 10.1109/MSP.2012.2187038

\section{About the Authors ...}

Jan ZAVRTÁLEK was born in Zlín, Czech Republic, in 1984. He received M.Sc. degrees from Czech Technical University in Prague (CTU) in Telecommunication Engineering and Radioelectronics in 2008, and from University of Economics, Prague in Econometrics and Operations Research in 2010. His specialization is electronic design and radioelectronics. He is currently working towards his Ph.D. degree at $\mathrm{CTU}$, focused on power line communication.

Daniel KEKRT was born in Prague, Czech Republic, in 1981. He received the Ph.D. degree from the Czech Technical University in Prague (CTU), Faculty of Electrical Engineering, in 2015. His research interests include image processing, iterative detection, 2D iterative decoding networks and joint iterative synchronization and detection.

Jaromír HRAD received the Ph.D. degree from the Czech Technical University in Prague (CTU), Faculty of Electrical Engineering, in 2003. His research interests include data transmission, power line communication, and also modern educational technologies. 